\section{A) Check for updates}

Cite this: Org. Biomol. Chem., 2019, 17,7192

Received 16th May 2019,

Accepted 2nd July 2019

DOI: $10.1039 / c 9 o b 01146 c$

rsc.li/obc

\title{
Visible light-mediated intermolecular [2 + 2] photocycloaddition of 1-aryl-2-nitroethenes and olefins $\uparrow$
}

\author{
Lisa-Marie Mohr, (D) Andreas Bauer, (iD Christian Jandl and Thorsten Bach (D) *
}

Despite the importance of cyclobutanes there are not many direct [2 +2 ] photocycloaddition reactions which can be performed with visible light in the absence of a catalyst. A notable exception is the reaction of 1-aryl-2-nitroethenes and olefins which can be performed at a wavelength of $\lambda=419 \mathrm{~nm}$ or $\lambda=$ $424 \mathrm{~nm}$ in $\mathrm{CH}_{2} \mathrm{Cl}_{2}$ as the solvent. In the present study, a total of 151 -aryl-2-nitroethenes were found to undergo a [2 + 2] photocycloaddition with 2,3-dimethyl-2-butene (28-86\% yield) and a set of 12 olefins was studied in their photocycloaddition to 1-phenyl-2-nitroethene (37-88\% yield). All mechanistic results are in agreement with a triplet reaction pathway and with the intermediacy of a 1,4-diradical.

\section{Introduction}

In 1887, when studying the properties of phenylnitroethylene ( $\beta$-nitrostyrene, 1-phenyl-2-nitroethene), Priebs observed that the yellow coloured substrate was converted upon exposure to light to a colourless product which he suspected to be a polymer. ${ }^{1}$ Meisenheimer and Heim reinvestigated the reaction in 1907 proposing a dimeric product in analogy to the photodimer of cinnamic acid. ${ }^{2}$ They were not able to prove this hypothesis, however, and it took another 65 years until the cyclobutane structure of the photodimer was established by Shechter and co-workers. ${ }^{3}$ The $[2+2]$ photodimerization was performed by exposure of solid trans- $\beta$-nitrostyrene to sunlight and the conversion was $c a$. 70\% after 4-6 weeks of irradiation. The relative configuration of the two diastereomeric products was later established by Desiraju and Pedireddi in an X-ray crystallographic study. ${ }^{4}$

Despite the fact that this precedence suggested that $\beta$-nitrostyrene can be involved in a $[2+2]$ photocycloaddition reaction when exposed to visible light, the very few attempts to obtain $[2+2]$ photocycloaddition products of $\beta$-nitrostyrene were performed with mercury lamps as UV irradiation sources. An initial report by Chapman and co-workers ${ }^{5}$ referred to work performed in the context of a Ph.D. thesis ${ }^{6}$ but did not provide

Department Chemie and Catalysis Research Center (CRC), Technische Universität München, 85747 Garching, Germany.E-mail: thorsten.bach@ch.tum.de;

Fax: +4989 28913315; Tel: +498928913330

$\dagger$ Electronic supplementary information (ESI) available: Synthetic procedures and full characterization for all starting materials (1) and products $(2,3,4,6,7)$, emission spectrum of 1a, quantum yield for 2a. CCDC 1915359. For ESI and crystallographic data in CIF or other electronic format see DOI: 10.1039/ C9OB01146C any experimental details. In 1980, the Sakurai group described the $[2+2]$ photocycloaddition of $\beta$-nitrostyrene with indene (Scheme 1) which was performed with a high-pressure mercury lamp in a pyrex vessel. ${ }^{7}$ Further photocycloaddition studies of 1-aryl-2-nitroethenes were reported by Ramkumar and Sankararaman (Michael type addition of silyl enol ethers to $\beta$-nitrostyrene $),{ }^{8}$ by Chapman and co-workers $([2+2]$ photocycloaddition of $\beta$-nitrostyrene and 2,3-dimethylbutadiene), ${ }^{9}$ and most recently by Ferreira and co-workers (Cr-catalysed $[4+2]$ cycloaddition of trans- $\beta$-nitro-para-methoxystyrene and 1,3-dienes). ${ }^{10}$

We became interested in the intermolecular $[2+2]$ photocycloaddition $^{11}$ of 1-aryl-2-nitroethenes in the context of our work on visible light-mediated reactions. ${ }^{12}$ In preliminary studies (Scheme 1), ${ }^{13}$ we found that a smooth reaction occurred when the title compounds $(c=20 \mathrm{mM})$ were irradiated in a solution of the olefin (10 equiv.) in dichloromethane at $\lambda=419 \mathrm{~nm}$. The reaction scope was limited,

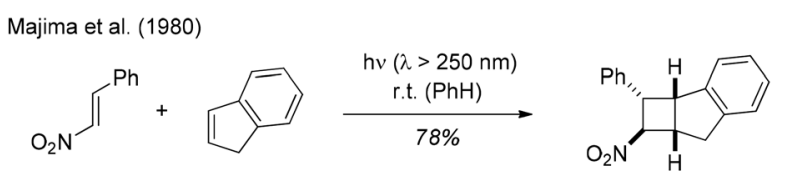

$$
\text { Mohr, Bach (2017) }
$$

Scheme 1 Previous studies on the title reaction. Visible light-mediated reactions were performed with fluorescent lamps (emission maximum: $\lambda=419 \mathrm{~nm})$. 
however, and the irradiation conditions were not fully optimized. We have now performed a more comprehensive array of experiments with a total number of 15 different 1-aryl-2nitroethenes and with an additional set of 12 olefins. Moreover, further mechanistic studies were performed to shed light on the course of the $[2+2]$ photocycloaddition. In this context, an unprecedented ring opening reaction of 1,1dicyclopropylethylene was observed. Full details of our experimental work are presented in this account.

\section{Reaction scope}

The 1-aryl-2-nitroethenes 1 employed in our study (Fig. 1) were prepared from nitromethane and the respective aromatic aldehydes in a Henry reaction. ${ }^{14}$ The condensation was performed with ammonium acetate in nitromethane or in a mixture of nitromethane and acetic acid. A colour change of the refluxing solution indicated a successful elimination of the intermediate alcohol to the nitroethene which was isolated exclusively as the trans-isomer. Only the 2-thiophenyl product 1e was not accessible by this method and required the use of a stronger base $(\mathrm{NaOH} \text { in } \mathrm{MeOH})^{15}$ to induce the Henry reaction.

$\mathrm{UV} /$ Vis-spectra ${ }^{16}$ of all 1-aryl-2-nitroethenes were recorded in dichloromethane solution and selected spectra are depicted in Fig. 2 (see the ESI† for all spectra). An electron withdrawing group at the phenyl group led to a hypsochromic shift relative to the parent compound 1a as shown for the 4-cyanophenyl derivative 1d. An electron donating group showed the opposite effect and the 4-methylphenyl (1b) and the 4-methoxyphenyl (1c) derivatives absorb at longer wavelength relative to 1a. The absorption coefficient is typically in the range of 10 000-20 000 $\mathrm{M}^{-1} \mathrm{~cm}^{-1}$ indicating that the absorption is due to an allowed transition (vide infra). All compounds are coloured which is in line with an - at least minimal - absorption in the visible range $(\lambda>380 \mathrm{~nm})$.

The previous preliminary irradiation experiments ${ }^{13}$ were conducted exclusively at room temperature with fluorescent lamps which display a relatively broad emission spectrum and an emission maximum at $\lambda=419 \mathrm{~nm}$ (Table 1 , conditions A).

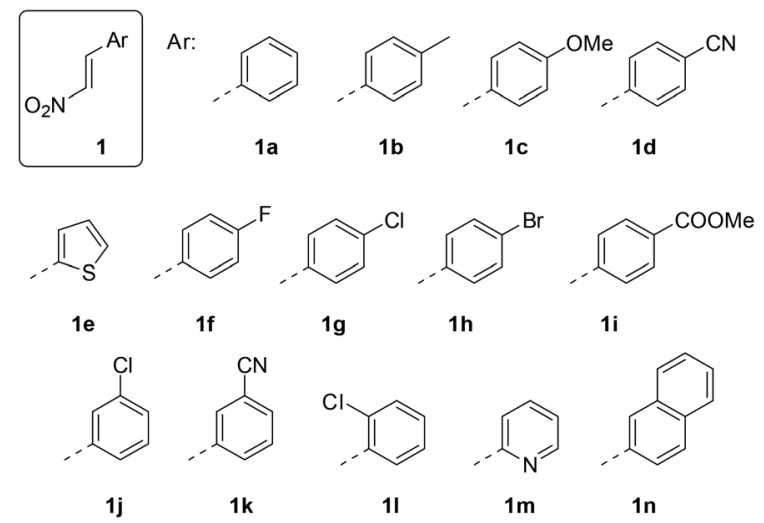

Fig. 1 Structures of the 1-aryl-2-nitroethenes 1 employed in this study.

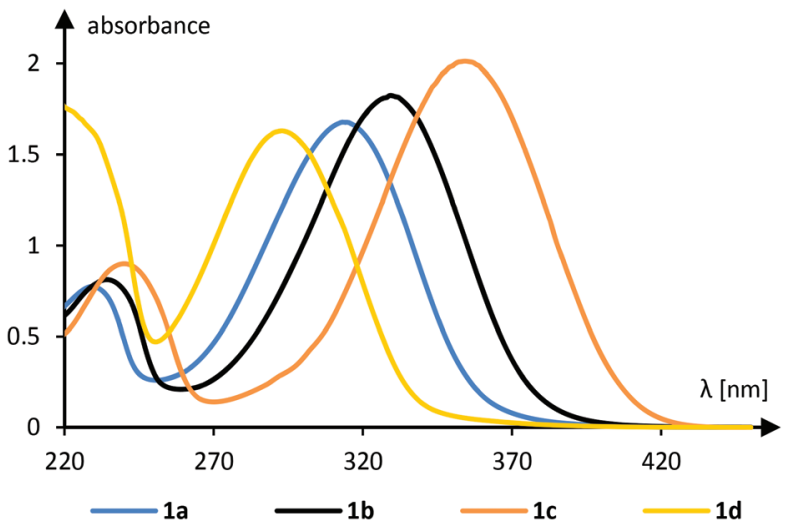

Fig. $2 \mathrm{UV} / \mathrm{Vis}$ spectra of selected 1-aryl-2-nitroethenes (c $=1 \mathrm{mM}$, $\mathrm{CH}_{2} \mathrm{Cl}_{2}$ ).

Table 1 Intermolecular $[2+2]$ photocycloaddition of 1-aryl-2nitroethenes (1) and 2,3-dimethyl-2-butene: optimal reaction conditions for the individual substrates

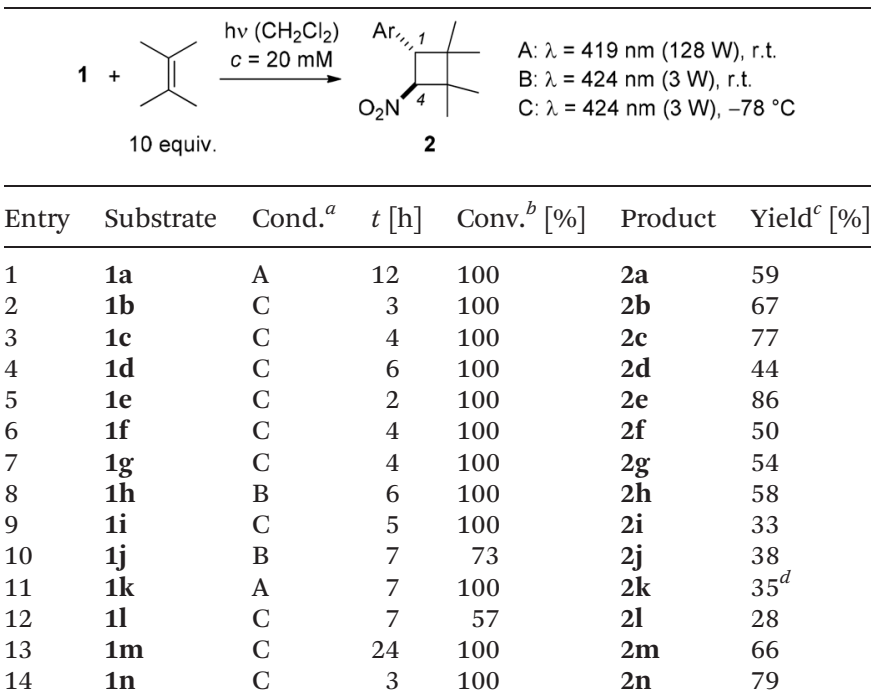

${ }^{a}$ The reactions were performed under conditions A, B, and C (see ESI $\dagger$ for further details). For each reaction the best conditions are listed in the table. Irradiation was discontinued after the indicated time period $t .{ }^{b}$ The conversion is based on the amount of re-isolated starting material. ${ }^{c}$ Yield of isolated product. ${ }^{d}$ Olefinic by-product $(11 \%)$, see narrative.

In search for optimal conditions, we also performed the reaction with a light-emitting diode (LED) at $\lambda=424 \mathrm{~nm}$ at ambient temperature (conditions B) and at $-78{ }^{\circ} \mathrm{C}$ (conditions $\mathrm{C}$ ). Every substrate was tested under all conditions in its reaction with 2,3-dimethyl-2-butene and the best conditions for the individual substrate are recorded in Table 1 (for the complete set of results see the $\mathrm{ESI}^{\dagger}$ ).

Unfunctionalized and heteroaromatic substrates (entries 1, $2,5,13$ and 14) reacted consistently well and in good yields (59-86\%). Methoxy and halogen substitution in para-position of the 1-phenyl-2-nitroethenes was compatible with the reaction (entries 3 and 6-8) and the respective products $2 \mathbf{c}, \mathbf{2 f}-\mathbf{2 h}$ 
were obtained in moderate to good yields $(50-77 \%)$. An electron withdrawing group (entries 4,9 and 11) retarded the reaction slightly which reflects a smaller absorption cross section of the substrates in the visible range ( $c f$. compound $\mathbf{1 d}$ in Fig. 2). In addition, side reactions were observed which were particularly significant for compound $\mathbf{1 k}$ (entry 11) and which will be discussed in the mechanistic section. The reactions of the meta- and ortho-chloro substituted 1-phenyl-2-nitroethenes (entries 10 and 12) proceeded sluggishly and were stopped after seven hours. Starting material was recovered as a mixture of the respective cis- and trans-compound. Likewise, whenever a reaction was stopped before completion, the recovered 1-aryl2-nitroethenes were isolated as cis-/trans-mixtures. The composition in the photostationary state reflects the different absorption properties of the individual geometric isomers at the chosen irradiation wavelength. ${ }^{13,17}$ The only substrate which did not show any $[2+2]$ photocycloaddition reaction was 1-(4'$\mathrm{N}, \mathrm{N}$-dimethylamino)phenyl-2-nitroethene despite the fact that it displays a particularly extensive absorption in the visible region. There was no decomposition of starting material and it is likely that intramolecular relaxation pathways ${ }^{18}$ occur more rapidly than the intermolecular addition to the olefin. Products 2 were isolated as single diastereoisomers with the aryl group (Ar) and the nitro group in trans-position at the cyclobutane ring. This assignment was corroborated by NOE experiments which revealed a contact between the ortho protons at the $\mathrm{C} 1$ phenyl group and the proton at $\mathrm{C} 4$. It is also in line with the relative configuration found in previously reported $[2+2]$ photocycloaddition products of trans$\beta$-nitrostyrene (1a). ${ }^{7,9}$

In our preliminary communication, the reaction of trans$\beta$-nitrostyrene (1a) with indene, vinyl ethyl ether, 2,3-dimethylbutadiene, and cyclopentene under visible light irradiation (conditions A) was reported. ${ }^{13}$ Scheme 2 displays reactions of

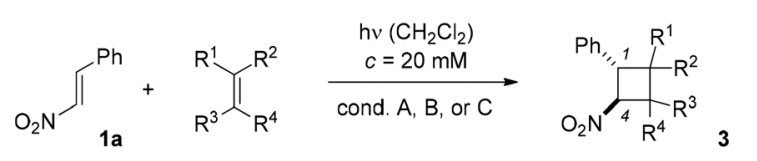

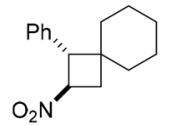
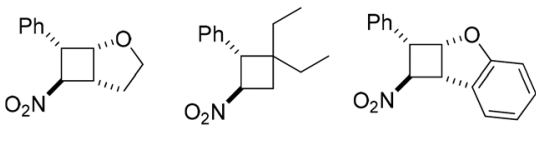

$3 \mathrm{a}(61 \%)$
$\mathrm{C}, t=14 \mathrm{~h}$

3b $(37 \%)$

$3 c(43 \%)$

$3 d(48 \%)$
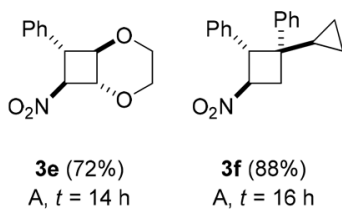

d.r. $=52 / 29 / 19$

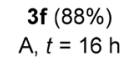

A, $t=16 h$

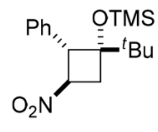

$3 g(38 \%)$

$\mathrm{B}, t=24 \mathrm{~h}$

d.r. $=77 / 23$

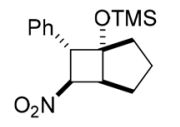

$3 \mathrm{~h}(38 \%)$

C, $t=24 \mathrm{~h}$

d.r. $=61 / 39$
Scheme 2 Intermolecular [2 + 2] photocycloaddition of 1-phenyl-2nitroethene (1a) and various olefins: optimal reaction conditions for the individual olefins ( $t=$ reaction time, $\mathrm{dr}=$ diastereomeric ratio). substrate 1a with olefins that had not been studied in previous work or that gave better yields under conditions B and C. Products $\mathbf{3 a - 3 d}$ were obtained as single isomers while cyclobutanes $3 \mathbf{e}-\mathbf{3 h}$ were formed as diastereomeric mixtures. It was possible in all cases to isolate the major isomer and to assign its relative configuration (see ESI $\uparrow$ for further details). The given yield refers to the total yield of all diastereoisomers $(\mathrm{dr}=$ diastereomeric ratio).

Electron deficient olefins (e.g. 1,1-dichloroethene, methyl acrylate, allylic alcohol) showed no reaction in attempted intermolecular $[2+2]$ photocycloaddition reactions with trans$\beta$-nitrostyrene (1a). In the reaction to product $\mathbf{3 f}$ there was no indication for a ring opening of the cyclopropyl ring and seven-membered carbocyclic by-products were not detected. The fact that silyl enol ethers gave cyclobutanes $\mathbf{3} \mathbf{g}$ and $\mathbf{3 h}$ as the only isolable products was surprising. In previous photochemical studies, ${ }^{8}$ Michael addition products were observed suggesting an addition reaction of the silyl enol ether with opposite regioselectivity. For comparison, we prepared the Michael addition product of 1-(trimethylsilyloxy)cyclopentene and trans- $\beta$-nitrostyrene by a thermal reaction. ${ }^{19}$ However, this very same product was not detectable in the crude product mixture of the $[2+2]$ photocycloaddition reaction neither by TLC nor by GLC analysis. It should be noted that different irradiation conditions $(\lambda>250 \mathrm{~nm})$ and a different substrate stoichiometry (1a: silyl enol ether $=1: 1)$ were used by Ramkumar and Sankararaman in their experiments. ${ }^{8}$ Still, it remains open why the regioselectivity should be completely reverted (vide infra). In all [2+2] photocycloaddition products 3 the better donor substituent of the former olefin is positioned at carbon atom $\mathrm{C} 2$ relative to carbon atom $\mathrm{C} 4$ which carries the nitro group.

Intramolecular $[2+2]$ photocycloaddition reactions were attempted with 1-phenyl-2-nitroethenes that had an alkenyl group linked to the ortho position of the phenyl ring, such as substrate $10^{20}$ (Scheme 3). Irrespective of the length of the tether there was no indication for an intramolecular reaction which could be due to the intrinsically low reactivity of a terminal olefin. An alternative explanation would be that initial C-C bond formation has to occur in the intramolecular reaction at the $\beta$-position of the nitrostyrene which might be electronically disfavored (vide infra). The chromophore of $\mathbf{1 0}$ is still reactive upon excitation as demonstrated by the intermolecular $[2+2]$ photocycloaddition of 2,3-dimethyl-2-butene to product $2 \mathbf{2}$.

Although synthetic applications of the nitrocyclobutanes were not in the focus of our current study, it was probed whether aminocyclobutanes would be accessible by a straight-

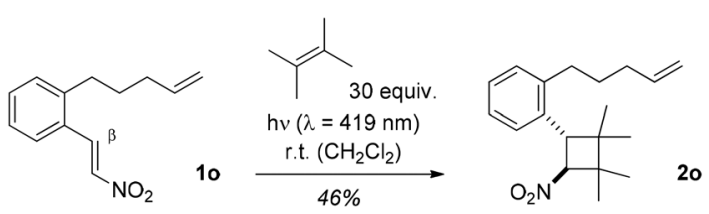

Scheme 3 Inter- vs. intramolecular [2 + 2] photocycloaddition of 1-aryl-2-nitroethene 10: exclusive formation of product 20 . 


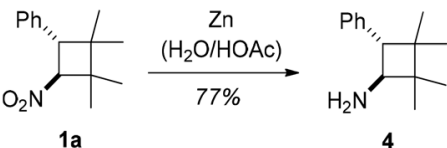

Scheme 4 Reduction of nitrocyclobutane 1a to aminocyclobutane 4.

forward reduction. ${ }^{21}$ Gratifyingly, the reduction of nitrocyclobutane 1a, as a representative example, with zinc $^{22}$ proceeded smoothly and without any loss of the stereochemical information. Product 4 was obtained in $77 \%$ yield (Scheme 4 ).

\section{Mechanistic studies}

There is consensus in the literature that the longest wavelength absorption of 1-aryl-2-nitroethenes correlates to a $\pi \pi^{*}$ transition into the respective first excited singlet state $\left(S_{1}\right) \cdot{ }^{16,18}$ For trans- $\beta$-nitrostyrene (1a), calculations have been performed that allow to visualize the electron density in the highest occupied molecular orbital (HOMO) and the lowest unoccupied molecular orbital (LUMO). ${ }^{23}$ There is no detectable fluorescence for typical 1-aryl-2-nitroethenes if the energy of $S_{1}$ is above $2.95 \mathrm{eV}\left(285 \mathrm{~kJ} \mathrm{~mol}^{-1}\right){ }^{16 b}$ Among other arguments, the fact, that fluorescence is not even observed in the solid state, suggests a rapid intersystem crossing (ISC) from the singlet to the triplet hypersurface leading to a population of the lowest lying triplet state $\left(\mathrm{T}_{1}\right)$. Only 1-phenyl-2-nitroethenes with a strong electron donating group (e.g. $\mathrm{NMe}_{2}$ ) exhibit fluorescence with a fluorescence quantum yield of $c a .0 .1$ in benzene. ${ }^{18}$ The photophysical behaviour of $1-\left(4^{\prime}-N, N\right.$-dimethylamino)phenyl-2-nitroethene has been studied by transient absorption spectroscopy. The ISC is extremely rapid $\left[\tau\left(\mathrm{S}_{1}\right) \cong\right.$ $6 \mathrm{ps}$ ] in a non-polar solvent (cyclohexane) and remains fast $\left[\tau\left(\mathrm{S}_{1}\right) \cong 70 \mathrm{ps}\right]$ in a solvent of moderate polarity. ${ }^{18}$

The triplet energies of compounds $\mathbf{1 a}, \mathbf{1 c}$, and $\mathbf{1 g}$ have been determined from their phosphorescence emission at $77 \mathrm{~K}$ in an EtOH matrix to be $E\left(\mathrm{~T}_{1}\right)=228 \mathrm{~kJ} \mathrm{~mol}^{-1}, 226 \mathrm{~kJ} \mathrm{~mol}^{-1}$, and $219 \mathrm{~kJ} \mathrm{~mol}^{-1}$, respectively. ${ }^{16 b}$ We recorded the phosphorescence spectrum of compound 1a in an EtOH matrix at $77 \mathrm{~K}$ and obtained a value of $E\left(\mathrm{~T}_{1}\right)=229 \mathrm{~kJ} \mathrm{~mol}^{-1}$ (see ESI $\dagger$ ). The nature of the triplet state for compounds $\mathbf{1}$ has not been extensively explored. Cowley assigned to it an $n \pi^{*}$ character which would be in accord with the high ISC rate and with the absence of fluorescence from $\mathrm{S}_{1}{ }^{16 b}$

Our mechanistic suggestion (Scheme 5) for the reaction course involves the $n \pi^{*}$ triplet state $\mathbf{1}\left(\mathrm{T}_{1}\right)$ as the key intermediate which is accessed from $1\left(\mathrm{~S}_{1}\right)$ by ISC. Electron loss at the oxygen $n$ orbitals and population of the $\pi^{*}$ orbital with an electron leads to electron deficiency at the $\alpha$-carbon atom (photochemical Umpolung) which is the preferred position of olefin attack to generate triplet diradical ${ }^{3} \mathbf{D}$. ISC and subsequent ring closure lead to cyclobutane products but side reactions are possible from ${ }^{3} \mathbf{D}$ prior or after ISC.

Any detectable side reactions which occur from ${ }^{3} \mathbf{D}$ support a pathway on the triplet hypersurface. As in our preliminary

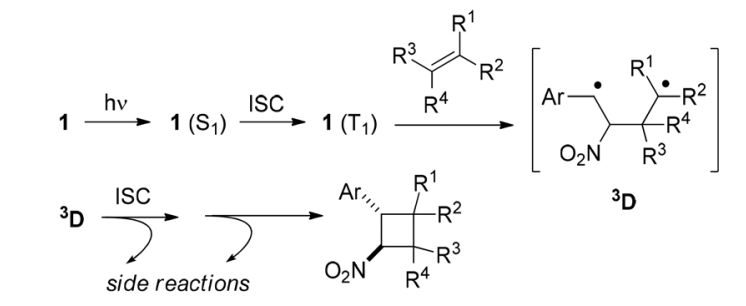

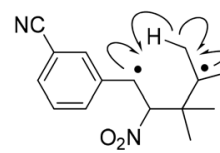

5

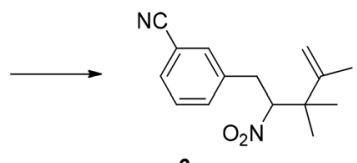

6
Scheme 5 Suggested reaction course of the [2+2] photocycloaddition between 1-aryl-2-nitroethenes and olefins via triplet 1,4 -diradical ${ }^{3} \mathrm{D}$ (top); formation of by-product 6 (Table 1, entry 11) via 1,4-diradical 5 (bottom).

studies, there were again products of a photo-ene reaction ${ }^{24}$ isolated as by-products. In the present study, the reaction of substrate $\mathbf{1 k}$ (Table 1 , entry 11) turned out to be particularly prone to undergo the reaction that likely involves an intramolecular hydrogen abstraction in 1,4-diradical 5 thus generating product 6.

Another way to substantiate the existence of a 1,4-diradical ${ }^{3} \mathbf{D}$ is based on the ring opening of a cyclopropyl-substituted alkyl radical. ${ }^{25}$ In the reaction to product $\mathbf{3 f}$, there was no indication for such a process, but when employing 1,1-dicyclopropylethylene $^{26}$ as substrate a new product was isolated apart from the regular $[2+2]$ photocycloaddition product $3 \mathbf{i}$. Proof for its tricyclic structure 7 rests - apart from extensive NMR analysis - on the isolation of the related product $\mathbf{8}$ from the reaction between 1-(4'-methoxycarbonyl)phenyl-2-nitroethene (1i) and 1,1-dicyclopropylethylene (Scheme 6).

$\mathrm{X}$-Ray crystallographic analysis of product 8 (Fig. 3) revealed the fact that both cyclopropyl rings had opened in the reaction sequence and that the exocyclic double bond was formally
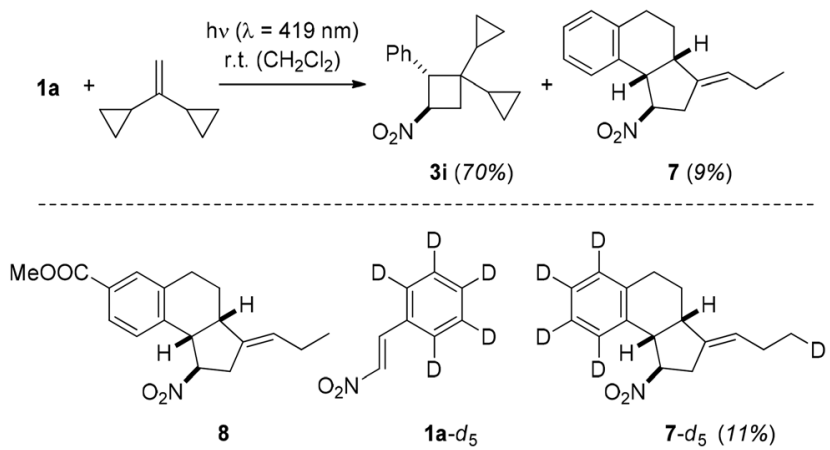

Scheme 6 Intermolecular $[2+2]$ photocycloaddition of 1-phenyl-2nitroethene (1a) and 1,1-dicyclopropylethylene to product $3 \mathbf{i}$ and byproduct 7 (top); by-products 8 obtained from 1-(4'-methoxycarbonyl) phenyl-2-nitroethene and 7- $d_{5}$ obtained from deuterated substrate 1a$d_{5}$ (bottom). 


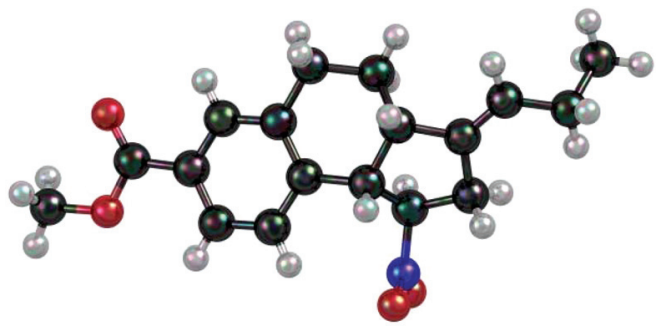

Fig. 3 Structure of by-product 8 as obtained by single-crystal X-ray diffraction.

$(E)$-configured. In order to explore the fate of the hydrogen atom in the ortho position of the phenyl ring, which is involved in a $\mathrm{C}-\mathrm{C}$ bond formation step, we submitted aldehyde $\mathbf{1 a}-\boldsymbol{d}_{\mathbf{5}}$ to the $[2+2]$ photocycloaddition with 1,1-dicyclopropylethylene (Scheme 6). Product $7-\boldsymbol{d}_{5}$ was isolated as by-product $(11 \%)$ together with the major product $3 \mathbf{i}-\boldsymbol{d}_{\mathbf{5}}(61 \%)$. The deuterium atom was found at the terminal carbon atom of the ethyl group which is attached to the exocyclic $(E)$-double bond.

Invoking a 1,4-diradical 9 for the reaction of $1 \mathrm{a}$ and 1,1dicyclopropylethylene the formation of 7 can be tentatively explained by the reaction cascade depicted in Scheme 7. Ring opening of the cyclopropane leads to 1,7-diradical 10 which seems unsuited for seven-membered ring formation. Instead, the radical in $\alpha$-position to the phenyl group attacks the double bond to produce 1,4-diradical 11 which opens to 1,7diradical 12. The proximity of the primary radical center to the phenyl ring in this intermediate may initiate a stereoselective $\mathrm{C}-\mathrm{C}$ bond formation with the former ortho hydrogen atom now being perfectly exposed for an intramolecular hydrogen abstraction. Indeed, molecular models suggest that this process is feasible in intermediate $\mathbf{1 3}$ leading to product 7.

The efficiency of the intermolecular $[2+2]$ photocycloaddition is limited not only by the low absorption coefficient of the 1-aryl-2-nitroethenes but also by their rapid cis/trans isomerisation in the excited state. ${ }^{18}$ The quantum yield for the reaction $\mathbf{1 a} \rightarrow \mathbf{2 a}$ at $\lambda=382 \mathrm{~nm}$ was determined as 0.04 (see ESI $\dagger$ for
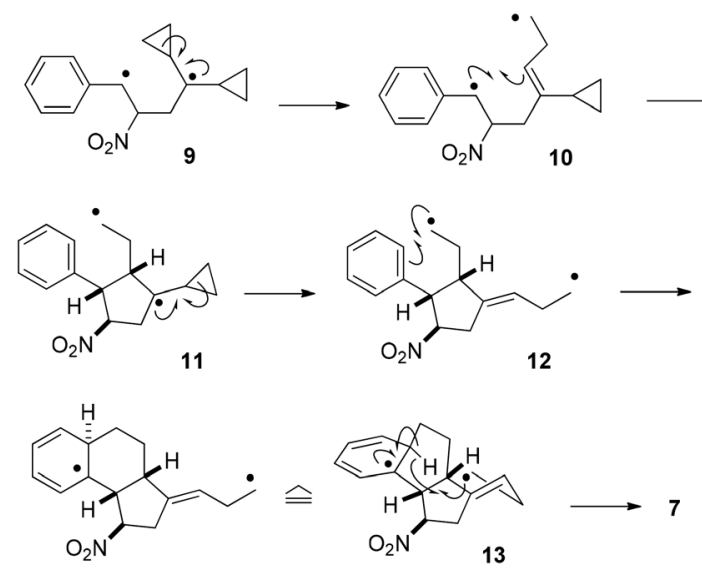

Scheme 7 Suggested formation of by-product 7 from 1,4-diradical intermediate 9 .

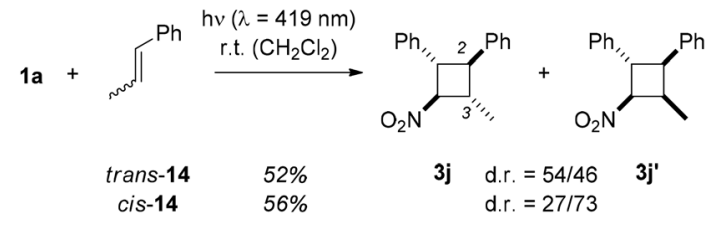

Scheme 8 Intermolecular [2 + 2] photocycloaddition of 1-phenyl-2nitroethene (1a) and $\beta$-methylstyrene (14): non-stereospecific reaction course, but incomplete stereoconvergence.

further details). Due to the rapid isomerisation it is experimentally difficult to determine whether both isomers are involved in the $[2+2]$ photocycloaddition but it is likely. Regarding the olefin, the stereochemical integrity during the $[2+2]$ photocycloaddition is high. Reactions performed with either trans- or cis- $\beta$-methyl styrene (14, Scheme 8) delivered the cyclobutanes $\mathbf{3} \mathbf{j}$ and $\mathbf{3} \mathbf{j}$ '. The recovered olefin was still diastereomerically pure in either case which indicates that the olefin is not photochemically excited under the irradiation conditions.

The photocycloaddition of olefins 14 was not stereospecific regarding the olefinic double bond. Starting from trans-14 significant amounts of product $\mathbf{3} \mathbf{j}^{\prime}$ were obtained with the two substituents at $\mathrm{C} 2$ and $\mathrm{C} 3$ in the cis-position. Vice versa, cis$\beta$-methyl styrene (cis-14) gave also notable quantities of the 2,3trans-product $\mathbf{3 j}$. In the absence of any detectable cis/trans isomerisation of $\beta$-methyl styrene during the reaction the nonstereospecifity is further evidence for the intermediacy of a triplet 1,4-diradical ${ }^{3} \mathbf{D}$ in which rotation around single bonds in possible. ${ }^{27}$

A final comment is warranted on a possible involvement of single electron transfer (SET) processes. The redox potential of trans- $\beta$-nitrostyrene (1a) in its triplet state can be estimated by its triplet state energy $E\left(\mathrm{~T}_{1}\right)=229 \mathrm{~kJ} \mathrm{~mol}^{-1}$ and by its ground state redox potential. ${ }^{28}$ Based on the known redox potential $E_{1 / 2}\left(\mathbf{1 a} / \mathbf{1 a}^{\cdot-}\right)=-0.44 \mathrm{~V}$ (ref. 29) a calculated value $E_{1 / 2}\left(\mathbf{1 a}^{*} / \mathbf{1 a}^{\cdot-}\right)$ in the order of $+1.90 \mathrm{~V}$ is obtained for the triplet state. Thermodynamically, the oxidation of several electron rich olefins with $E_{\text {ox }}\left(\right.$ olefin ${ }^{++} /$olefin $)<+1.90 \mathrm{~V}$ would thus seem feasible, e.g. of 2,3-dimethyl-2-butene $\left(E_{\text {ox }}=+1.50 \mathrm{~V}\right),{ }^{30} 2,3-$ dihydrofuran $\left(E_{\mathrm{ox}}=+1.40 \mathrm{~V}\right),{ }^{31}$ and 1-tert-butyl-1-(trimethylsilyloxy)ethene $\left(E_{\mathrm{ox}}=+1.34 \mathrm{~V}\right) .{ }^{32}$ However, several other reactive olefins, e.g. methylenecyclohexane $\left(E_{\mathrm{ox}}=+2.62 \mathrm{~V}\right),{ }^{33}$ exhibit a redox potential far too high for an electron transfer to be possible. In addition, SET reactions ${ }^{34}$ are typically performed in polar solvents to assist charge separation which is more difficult in a nonpolar solvent. The fact that the observed photocycloaddition works also in benzene and that it is accelerated by a triplet sensitizer ${ }^{13}$ makes the involvement of SET processes unlikely. Further circumstantial evidence is based on the absence of by-products which would be expected in the reaction of dienes $([4+2]$ cycloaddition $)$ and of 2,3-dimethyl-2butene. The side products mentioned earlier (e.g. product 6 , Scheme 5) should exhibit a different regioselectivity of addition $^{35}$ had they been formed in an SET process. 


\section{Conclusions}

To summarize, we have established a straightforward access to various 1-aryl-4-nitrocyclobutanes by a visible light-mediated intermolecular $[2+2]$ photocycloaddition. The substituents are trans-positioned within the cyclobutane but it is known that the relative configuration can be inverted to cis by a deprotonation/protonation sequence. ${ }^{3}$ In addition, the nitro group can be interconverted to an amine if desired as has been shown in the present study by reduction to product 4 . Accordingly, the method makes also trans- and cis-1-aryl-4aminocyclobutanes accessible if desired.

Although the nitro chromophore bears electronically some analogy to a carbonyl group, the photochemical behaviour of 1-phenyl-2-nitroethene is different from cinnamic aldehyde. While the latter compound does not form cyclobutanes upon direct irradiation ${ }^{36}$ the former compound and its analogues are suitable substrates for [2+2] photocycloaddition reactions, as shown in this study. For 1-aryl-2-nitroethenes, an intrinsic feature of their excited state seems to be the propensity to react only with electron rich olefins.

\section{Experimental}

\section{General information}

All preparations and manipulations of air and moisture sensitive compounds were carried out in flame dried glassware under an argon atmosphere using standard Schlenk techniques. Dry solvents were either obtained water and oxygen free by a Braun MB SPS purification system or from commercial sources (see ESI $\dagger$ ). Irradiation experiments were either performed in a Rayonet RPR-100 photochemical reactor (Southern New England Ultra Violet Company, Branford, CT, USA) at $\lambda_{\max }=419 \mathrm{~nm}$ (16 lamps, cool white, $8 \mathrm{~W}$, Osram $)^{37}$ or with a high power light emitting diode (LED) at $\lambda_{\max }=$ $424 \mathrm{~nm}$ (Roithner Lasertechnik, $350 \mathrm{~mA}$, UF $3.4 \mathrm{~V}$ ). Flash column chromatography was performed with silica 60 (Merck, 230-400 mesh) as the stationary phase. Infrared (IR) spectra were recorded on a PerkinElmer Frontier IR FTR spectrometer by ATR technique. Nuclear magnetic resonance (NMR) spectra were recorded at room temperature either on a Bruker AVHD 300, AVHD 400, AVHD 500 or an AV 500 cryo. ${ }^{1} \mathrm{H}$ NMR spectra were referenced to the residual proton signal of the respective solvent. ${ }^{13} \mathrm{C}$ NMR spectra were referenced to the ${ }^{13} \mathrm{C}$-D multiplet of the solvent employed. Assignment and multiplicity of the ${ }^{13} \mathrm{C}$ NMR signals were determined by twodimensional NMR experiments (COSY, HSQC, HMBC). The relative configuration of diastereoisomers was corroborated by NOESY. Melting points were determined using a Kofler melting point apparatus ("Thermopan", Reichert), with a range quoted to the nearest whole number. Mass spectrometry (MS) was performed on a GC-coupled Agilent system (EI, $70 \mathrm{eV}$ ). High resolution mass spectrometry (HRMS) was performed on a Thermo Scientific LTQ FT Ultra (ESI) or a Thermo Scientific DFS HRMS spectrometer (EI). UV/Vis spectra were measured on a PerkinElmer Lambda 35 UV/Vis spectrometer.

\section{Experimental Procedures}

$\left(2^{\prime}, 2^{\prime}, 3^{\prime}, 3^{\prime}\right.$-Tetramethyl-4'-nitrocyclobutyl)-benzene (2a). Representative procedure (conditions A): A solution of nitroethene 1a (29.8 mg, $200 \mu \mathrm{mol}, 1.00$ equiv.) and 2,3-dimethyl-2-butene ( $234 \mu \mathrm{L}, 166 \mathrm{mg}, 2.00 \mathrm{mmol}, 10.0$ equiv.) in dichloromethane $(10 \mathrm{~mL}, c=20 \mathrm{mM})$ was irradiated at $\lambda_{\max }=419 \mathrm{~nm}$ for twelve hours at room temperature. The crude product was purified by column chromatography $\left(\mathrm{P} / \mathrm{Et}_{2} \mathrm{O}=20 / 1\right)$ to yield $2 \mathrm{a}(26.9 \mathrm{mg}, 1.16 \mathrm{mmol}, 59 \%)$ as a yellow coloured oil. The analytical data obtained matched those reported in the literature. ${ }^{13}$

1-Methyl-4-(2',2',3', 3'-tetramethyl-4'-nitrocyclobutyl)-benzene (2b). Representative procedure (conditions C): A solution of nitroethene $\mathbf{1 b}(16.3 \mathrm{mg}, 100 \mu \mathrm{mol}, 1.00$ equiv.) and 2,3-dimethyl-2-butene ( $119 \mu \mathrm{L}, 84.1 \mathrm{mg}, 1.00 \mathrm{mmol}, 10.0$ equiv.) in dichloromethane $(5 \mathrm{~mL}, c=20 \mathrm{~mm})$ was irradiated at $\lambda_{\max }=$ $424 \mathrm{~nm}$ for three hours at $-78^{\circ} \mathrm{C}$. The crude product was purified by column chromatography $\left(\mathrm{P} / \mathrm{Et}_{2} \mathrm{O}=20 / 1\right)$ to yield $2 \mathbf{b}$ (16.5 mg, $66.7 \mu \mathrm{mol}, 67 \%$ ) as a pale-yellow coloured oil. The analytical data obtained matched those reported in the literature. $^{13}$

1-Methoxy-4-(2',2',3',3'-tetramethyl-4'-nitrocyclobutyl)-benzene (2c). The reaction was performed in analogy to the representative procedure for conditions $\mathrm{C}$ (see above) with nitroethene $\mathbf{1 c}$ (17.9 mg, $100 \mu \mathrm{mol}, 1.00$ equiv.) and 2,3-dimethyl-2-butene (119 $\mu \mathrm{L}, 84.1 \mathrm{mg}, 1.00 \mathrm{mmol}, 10.0$ equiv.) in dichloromethane ( $5 \mathrm{~mL}, c=20 \mathrm{~mm})(t=4 \mathrm{~h})$. Purification by column chromatography $\left(\mathrm{P} / \mathrm{Et}_{2} \mathrm{O}=9 / 1\right)$ yielded $2 \mathrm{c}(20.3 \mathrm{mg}, 77.1 \mu \mathrm{mol}, 77 \%)$ as a yellow coloured oil. The analytical data obtained matched those reported in the literature. ${ }^{13}$

4-(2',2',3',3'-Tetramethyl-4'-nitrocyclobutyl)-benzonitrile (2d). The reaction was performed in analogy to the representative procedure for conditions $\mathrm{C}$ (see above) with nitroethene 1d (17.4 mg, $100 \mu \mathrm{mol}, 1.00$ equiv.) and 2,3-dimethyl-2-butene (119 $\mu \mathrm{L}, 84.1 \mathrm{mg}, 1.00 \mathrm{mmol}, 10.0$ equiv.) in dichloromethane ( $5 \mathrm{~mL}, c=20 \mathrm{~mm})(t=6 \mathrm{~h})$. Purification by column chromatography $\left(\mathrm{P} / \mathrm{Et}_{2} \mathrm{O}=9 / 1\right)$ yielded $2 \mathrm{~d}(11.4 \mathrm{mg}, 43.9 \mu \mathrm{mol}, 44 \%)$ as colourless solid. The by-product was only observed in traces. The analytical data obtained matched those reported in the literature. $^{13}$

2-(2', $2^{\prime}, 3^{\prime}, 3^{\prime}$-Tetramethyl-4'-nitrocyclobutyl)-thiophene (2e). The reaction was performed in analogy to the representative procedure for conditions $\mathrm{C}$ (see above) with nitroethene 1e (15.5 mg, $100 \mu \mathrm{mol}, 1.00$ equiv.) and 2,3-dimethyl-2-butene (119 $\mu \mathrm{L}, 84.1 \mathrm{mg}, 1.00 \mathrm{mmol}, 10.0$ equiv.) in dichloromethane ( $5 \mathrm{~mL}, c=20 \mathrm{~mm})(t=2 \mathrm{~h})$. Purification by column chromatography $\left(\mathrm{P} / \mathrm{Et}_{2} \mathrm{O}=19 / 1\right)$ yielded $2 \mathrm{e}(20.5 \mathrm{mg}, 85.7 \mu \mathrm{mol}, 86 \%)$ as a yellow coloured oil. The analytical data obtained matched those reported in the literature. ${ }^{13}$

1-Fluoro-4-(2', 2',3',3'-tetramethyl-4'-nitrocyclobutyl)-benzene (2f). The reaction was performed in analogy to the representative procedure for conditions $\mathrm{C}$ (see above) with nitroethene $\mathbf{1 f}$ (16.7 mg, $100 \mu \mathrm{mol}, 1.00$ equiv.) and 2,3-dimethyl-2-butene 
(119 $\mu \mathrm{L}, 84.1 \mathrm{mg}, 1.00 \mathrm{mmol}, 10.0$ equiv.) in dichloromethane ( $5 \mathrm{~mL}, c=20 \mathrm{~mm})(t=4 \mathrm{~h})$. Purification by column chromatography $\left(\mathrm{P} / \mathrm{Et}_{2} \mathrm{O}=15 / 1\right)$ yielded $2 \mathrm{f}(12.6 \mathrm{mg}, 50.1 \mu \mathrm{mol}, 50 \%)$ as a colourless solid. $R_{\mathrm{f}}=0.53\left(\mathrm{P} / \mathrm{Et}_{2} \mathrm{O}=9 / 1\right)$; IR: $\tilde{\nu}\left(\mathrm{cm}^{-1}\right)=3079$, 2967, 2871, 1538, 1510, 1460, 1371, 1226, 1151, 1135, 844, 761; ${ }^{1} \mathrm{H}$ NMR $\left(400 \mathrm{MHz}, \mathrm{CDCl}_{3}\right): \delta(\mathrm{ppm})=7.09-7.00\left(\mathrm{~m}, 4 \mathrm{H}, \mathrm{H}_{\mathrm{ar}}\right)$, $4.85\left(\mathrm{~d},{ }^{3} J=10.1 \mathrm{~Hz}, 1 \mathrm{H}, \mathrm{H}-4^{\prime}\right), 3.92\left(\mathrm{~d},{ }^{3} J=10.1 \mathrm{~Hz}, 1 \mathrm{H}, \mathrm{H}-1^{\prime}\right)$, 1.24 (s, 3H, $\left.\mathrm{CH}_{3}-2^{\prime}\right), 1.16$ (s, 3H, $\left.\mathrm{CH}_{3}-3^{\prime}\right), 1.14$ (s, 3H, $\left.\mathrm{CH}_{3}-2^{\prime}\right)$, $0.70\left(\mathrm{~s}, 3 \mathrm{H}, \mathrm{CH}_{3}-3^{\prime}\right) ;{ }^{13} \mathrm{C} \mathrm{NMR}\left(101 \mathrm{MHz}, \mathrm{CDCl}_{3}\right): \delta(\mathrm{ppm})=$ $162.1\left(\mathrm{~d},{ }^{1} J_{\mathrm{CF}}=254.4 \mathrm{~Hz}, \mathrm{C}-1\right), 132.2(\mathrm{~s}, \mathrm{C}-4), 128.5\left(\mathrm{~d},{ }^{3} J_{\mathrm{CF}}=\right.$ $7.9 \mathrm{~Hz}, \mathrm{C}-3, \mathrm{C}-5), 115.6$ (d, $\left.{ }^{2} J_{\mathrm{CF}}=21.4 \mathrm{~Hz}, \mathrm{C}-2, \mathrm{C}-6\right), 85.2$ (d, C-4'), 48.9 (d, C-1'), 45.0 (s, C-3'), 39.3 (s, C-2'), 24.2 [q, (C-3') $\left.\mathrm{CH}_{3}\right], 22.8$ [q, $\left.\left(\mathrm{C}-2^{\prime}\right) \mathrm{CH}_{3}\right], 21.4$ [q, $\left.\left(\mathrm{C}-3^{\prime}\right) \mathrm{CH}_{3}\right], 19.4$ [q, (C-2') $\left.\mathrm{CH}_{3}\right] ; \mathrm{MS}$ (EI): $m / z(\%)=205$ (45) $\left[\mathrm{M}-\mathrm{NO}_{2}\right]^{+}, 163$ (100) $\left[\mathrm{M}-\mathrm{NO}_{2}-\mathrm{C}_{3} \mathrm{H}_{6}\right]^{+}, 106(54)\left[\mathrm{C}_{7} \mathrm{H}_{6} \mathrm{~F}\right]^{+}$; $\operatorname{HRMS}$ (EI, $\left.70 \mathrm{eV}\right)$ : calcd for $\mathrm{C}_{14} \mathrm{H}_{18} \mathrm{FNO}_{2}^{+}[\mathrm{M}]^{+}$: 251.1316; found: 251.1316.

1-Chloro-4-(2', 2', 3', 3'-tetramethyl-4'-nitrocyclobutyl)-benzene (2g). The reaction was performed in analogy to the representative procedure for conditions $\mathrm{C}$ (see above) with nitroethene $\mathbf{1 g}$ (18.3 mg, $100 \mu \mathrm{mol}, 1.00$ equiv.) and 2,3-dimethyl-2-butene $(119 \mu \mathrm{L}, 84.1 \mathrm{mg}, 1.00 \mathrm{mmol}, 10.0$ equiv.) in dichloromethane ( $5 \mathrm{~mL}, c=20 \mathrm{~mm}$ ) $(t=4 \mathrm{~h})$. Purification by column chromatography $\left(\mathrm{P} / \mathrm{Et}_{2} \mathrm{O}=19 / 1\right)$ yielded $2 \mathrm{~g}(14.4 \mathrm{mg}, 53.8 \mu \mathrm{mol}, 54 \%)$ as a pale yellow coloured solid. $R_{\mathrm{f}}=0.54\left(\mathrm{P} / \mathrm{Et}_{2} \mathrm{O}=9 / 1\right)$; IR: $\tilde{\nu}\left(\mathrm{cm}^{-1}\right)=3073,2972,2958,1539,1494,1370,1153,1135$, 1089, 877, 839, 767; ${ }^{1} \mathrm{H}$ NMR (400 $\left.\mathrm{MHz}, \mathrm{CDCl}_{3}\right): \delta(\mathrm{ppm})=$ 7.33-7.29 (m, 2H, H-2, H-6), 7.06-7.02 (m, 2H, H-3, H-5), 4.85 (d, $\left.{ }^{3} J=10.0 \mathrm{~Hz}, 1 \mathrm{H}, \mathrm{H}-4^{\prime}\right), 3.92\left(\mathrm{~d},{ }^{3} J=10.0 \mathrm{~Hz}, 1 \mathrm{H}, \mathrm{H}-1^{\prime}\right), 1.24$ (s, 3H, $\mathrm{CH}_{3}-2^{\prime}$ ), 1.17 (s, 3H, $\left.\mathrm{CH}_{3}-3^{\prime}\right), 1.15$ (s, 3H, $\left.\mathrm{CH}_{3}-2^{\prime}\right), 0.70$ $\left(\mathrm{s}, 3 \mathrm{H}, \mathrm{CH}_{3}-3^{\prime}\right) ;{ }^{13} \mathrm{C} \mathrm{NMR}\left(101 \mathrm{MHz}, \mathrm{CDCl}_{3}\right): \delta(\mathrm{ppm})=134.9(\mathrm{~s}$, C-4), 133.0 (s, C-1), 128.9 (d, 2C, C-2, C-6), 128.4 (d, 2C, C-3, C-5), 84.9 (d, C-4'), 49.0 (d, C-1'), 45.1 (s, C-3'), 39.4 (s, C-2'), 24.3 [q, $\left.\left(\mathrm{C}-3^{\prime}\right) \mathrm{CH}_{3}\right], 22.8$ [q, $\left.\left(\mathrm{C}-2^{\prime}\right) \mathrm{CH}_{3}\right], 21.5\left[\mathrm{q},\left(\mathrm{C}-3^{\prime}\right) \mathrm{CH}_{3}\right]$, 19.5 [q, (C-2') $\left.\mathrm{CH}_{3}\right]$; $\mathrm{MS}(\mathrm{EI}): m / z(\%)=221(29)\left[\mathrm{M}-\mathrm{NO}_{2}\right]^{+}, 179$ (100) $\left[\mathrm{M}-\mathrm{NO}_{2}-\mathrm{C}_{3} \mathrm{H}_{6}\right]^{+}, 125(64)\left[\mathrm{C}_{7} \mathrm{H}_{6} \mathrm{Cl}\right]^{+}$; HRMS (EI, $\left.70 \mathrm{eV}\right)$ : calcd for $\mathrm{C}_{14} \mathrm{H}_{18} \mathrm{ClNO}_{2}{ }^{+}[\mathrm{M}]^{+}$: 267.1021; found: 267.1021 .

1-Bromo-4-(2',2',3',3'-tetramethyl-4'-nitrocyclobutyl)-benzene (2h). Representative procedure (conditions B): A solution of nitroethene $\mathbf{1 h}$ (22.8 mg, $100 \mu \mathrm{mol}, 1.00$ equiv.) and 2,3dimethyl-2-butene (119 $\mu \mathrm{L}, 84.2 \mathrm{mg}, 1.00 \mathrm{mmol}, 10.0$ equiv.) in dichloromethane $(5 \mathrm{~mL}, c=20 \mathrm{~mm})$ was irradiated at $\lambda_{\max }=$ $424 \mathrm{~nm}$ for six hours at room temperature. The crude product was purified by column chromatography $\left(\mathrm{P} / \mathrm{Et}_{2} \mathrm{O}=20 / 1\right)$ to yield $2 \mathbf{h}(18.0 \mathrm{mg}, 56.0 \mu \mathrm{mol}, 58 \%)$ as a yellow coloured oil. $R_{\mathrm{f}}=0.43\left(\mathrm{P} / \mathrm{Et}_{2} \mathrm{O}=9 / 1\right)$; IR: $\tilde{\nu}\left(\mathrm{cm}^{-1}\right)=2962,2925,1552,1464$, 1376, 1259, 1072, 1010, 861, 797; ${ }^{1} \mathrm{H}$ NMR (500 MHz, $\mathrm{CDCl}_{3}$ ): $\delta[\mathrm{ppm}]=7.48-7.44(\mathrm{~m}, 2 \mathrm{H}, \mathrm{H}-2, \mathrm{H}-6), 7.00-6.96(\mathrm{~m}, 2 \mathrm{H}, \mathrm{H}-3$, $\mathrm{H}-5$ ), 4.85 (d, $\left.{ }^{3} J=10.1 \mathrm{~Hz}, 1 \mathrm{H}, \mathrm{H}-4^{\prime}\right), 3.90$ (d, ${ }^{3} J=10.1 \mathrm{~Hz}, 1 \mathrm{H}$, $\mathrm{H}-1^{\prime}$ ), 1.23 (s, 3H, $\left.\mathrm{CH}_{3}-3^{\prime}\right), 1.17$ (s, 3H, $\left.\mathrm{CH}_{3}-2^{\prime}\right), 1.14(\mathrm{~s}, 3 \mathrm{H}$, $\left.\mathrm{CH}_{3}-3^{\prime}\right), 0.70\left(\mathrm{~s}, 3 \mathrm{H}, \mathrm{CH}_{3}-2^{\prime}\right) ;{ }^{13} \mathrm{C} \mathrm{NMR}$ (101 $\mathrm{MHz}, \mathrm{CDCl}_{3}$ ): $\delta(\mathrm{ppm})=135.5(\mathrm{~s}, \mathrm{C}-1), 131.8(\mathrm{~d}, 2 \mathrm{C}, \mathrm{C}-2, \mathrm{C}-6), 128.7(\mathrm{~d}, 2 \mathrm{C}$, C-3, C-5), 121.1 (s, C-4), 84.8 (d, C-4'), 49.1 (d, C-1'), 45.1 (s, C-3'), 39.4 (s, C-2'), 24.3 [q, $\left(\mathrm{C}-3^{\prime}\right) \mathrm{CH}_{3}$ ], 22.8 [q, $\left.\left(\mathrm{C}-2^{\prime}\right) C \mathrm{H}_{3}\right], 21.5$ $\left[\mathrm{q},\left(\mathrm{C}-3^{\prime}\right) \mathrm{CH}_{3}\right], 19.5$ [q, $\left.\left(\mathrm{C}-2^{\prime}\right) \mathrm{CH}_{3}\right]$; MS (EI): $\mathrm{m} / z(\%)=265$ (20) $\left[\mathrm{M}-\mathrm{NO}_{2}\right]^{+}, 168$ (100) $\left[\mathrm{M}-\mathrm{NO}_{2}-\mathrm{Br}\right]^{+}, 143(56)\left[\mathrm{M}-\mathrm{NO}_{2}-\right.$ $\left.\mathrm{Br}-\mathrm{C}_{3} \mathrm{H}_{6}\right]^{+}$; HRMS (ESI): calcd for $\mathrm{C}_{14} \mathrm{H}_{19}{ }^{79} \mathrm{BrNO}_{2}{ }^{+}[\mathrm{M}+\mathrm{H}]^{+}$:
312.0594; found: 312.0593, calcd for $\mathrm{C}_{14} \mathrm{H}_{19}{ }^{81} \mathrm{BrNO}_{2}{ }^{+}[\mathrm{M}+\mathrm{H}]^{+}$: 314.0573; found: 314.0573 .

Methyl 4-(2',2',3',3'-tetramethyl-4'-nitrocyclobutyl)-benzoate (2i). The reaction was performed in analogy to the representative procedure for conditions $\mathrm{C}$ (see above) with nitroethene $\mathbf{1 i}$ (20.7 mg, $100 \mu \mathrm{mol}, 1.00$ equiv.) and 2,3-dimethyl-2-butene (119 $\mu \mathrm{L}, 84.1 \mathrm{mg}, 1.00 \mathrm{mmol}, 10.0$ equiv.) in dichloromethane ( $5 \mathrm{~mL}, c=20 \mathrm{~mm})(t=5 \mathrm{~h})$. Purification by column chromatography $\left(\mathrm{P} / \mathrm{Et}_{2} \mathrm{O}=9 / 1\right)$ yielded $2 \mathrm{i}(9.47 \mathrm{mg}, 32.5 \mu \mathrm{mol}, 33 \%)$ and a by-product (see ESI, $\uparrow 1.23 \mathrm{mg}, 4.22 \mu \mathrm{mol}, 4 \%$ ) as a mixture (colourless solid). $R_{\mathrm{f}}=0.64\left(\mathrm{P} / \mathrm{Et}_{2} \mathrm{O}=1 / 1\right)$; mp: $112{ }^{\circ} \mathrm{C}$; IR: $\tilde{\nu}\left(\mathrm{cm}^{-1}\right)=2954,1717,1541,1433,1371,1277,1181,1151$, 1138, 1110, 1017, 860, 756; ${ }^{1} \mathrm{H}$ NMR (400 $\left.\mathrm{MHz}, \mathrm{CDCl}_{3}\right)$ : $\delta(\mathrm{ppm})=8.00\left(\mathrm{~d},{ }^{3} J=7.3 \mathrm{~Hz}, 2 \mathrm{H}, \mathrm{H}-2, \mathrm{H}-6\right), 7.18\left(\mathrm{~d},{ }^{3} J=7.3\right.$ $\mathrm{Hz}, 2 \mathrm{H}, \mathrm{H}-3, \mathrm{H}-5), 4.92$ (d, $\left.{ }^{3} J=9.9 \mathrm{~Hz}, 1 \mathrm{H}, \mathrm{H}-4^{\prime}\right), 4.01$ (d, ${ }^{3} J=$ 9.9 Hz, 1H, H-1'), 1.24 (s, 3H, $\left.\mathrm{CH}_{3}-3^{\prime}\right), 1.20$ (s, 3H, $\left.\mathrm{CH}_{3}-2^{\prime}\right), 1.16$ (s, 3H, $\left.\mathrm{CH}_{3}-3^{\prime}\right), 0.69$ (s, 3H, $\left.\mathrm{CH}_{3}-2^{\prime}\right) ;{ }^{13} \mathrm{C} \mathrm{NMR}(101 \mathrm{MHz}$, $\left.\mathrm{CDCl}_{3}\right): \delta(\mathrm{ppm})=167.0\left(\mathrm{~s}, \mathrm{CH}_{3} \mathrm{CO}_{2} \mathrm{Ar}\right), 141.8(\mathrm{~s}, \mathrm{C}-1), 130.0$ (d, 2C, C-2, C-6), 129.1 (s, C-4), 127.0 (d, 2C, C-3, C-5), 84.6 (d, C-4'), 53.2 (q, $\left.\mathrm{CH}_{3} \mathrm{CO}_{2} \mathrm{Ar}\right) 49.5$ (d, C-1'), 45.1 (s, C-2'), 39.7 (s, C-3'), 24.3 [q, $\left(\mathrm{C}-3^{\prime}\right) \mathrm{CH}_{3}$ ], 22.7 [q, $\left.\left(\mathrm{C}-2^{\prime}\right) \mathrm{CH}_{3}\right], 21.5$ [q, (C-2') $\left.\mathrm{CH}_{3}\right], 19.5$ [q, $\left.\left.\left(\mathrm{C}-3^{\prime}\right) \mathrm{CH}_{3}\right] ; \mathrm{MS}(\mathrm{EI}): m / z \quad \%\right)=245$ (92) $\left[\mathrm{M}-\mathrm{NO}_{2}\right]^{+}, 203$ (39) $\left[\mathrm{M}-\mathrm{NO}_{2}-\mathrm{C}_{3} \mathrm{H}_{6}\right]^{+}, 171$ (51), 159 (34), 84 (100) $\left[\mathrm{C}_{6} \mathrm{H}_{12}\right]^{+}, 69$ (35); HRMS (ESI): calcd for $\mathrm{C}_{16} \mathrm{H}_{22} \mathrm{NO}_{4}{ }^{+}$ $[\mathrm{M}+\mathrm{H}]^{+}$292.1543; found: 292.1544.

1-Chloro-3-(2',2',3',3'-tetramethyl-4'-nitrocyclobutyl)-benzene $(2 \mathrm{j})$. The reaction was performed in analogy to the representative procedure for conditions $B$ (see above) with nitroethene $\mathbf{1 j}$ (18.4 mg, $100 \mu \mathrm{mol}, 1.00$ equiv.) and 2,3-dimethyl-2-butene (119 $\mu \mathrm{L}, 84.2 \mathrm{mg}, 1.00 \mathrm{mmol}, 10.0$ equiv.) in dichloromethane ( $5 \mathrm{~mL}, c=20 \mathrm{~mm})(t=7 \mathrm{~h})$. Purification by column chromatography $\left(\mathrm{P} / \mathrm{Et}_{2} \mathrm{O}=19 / 1\right)$ yielded $2 \mathbf{j}(10.3 \mathrm{mg}, 38.5 \mu \mathrm{mol}, 38 \%)$ as a colourless oil. Starting material was recovered as transisomer trans-1j (5.00 mg, $27.2 \mu \mathrm{mol}, 27 \%) . R_{\mathrm{f}}=0.53\left(\mathrm{P} / \mathrm{Et}_{2} \mathrm{O}=\right.$ 9/1); IR: $\tilde{\nu}\left(\mathrm{cm}^{-1}\right)=3066,2967,1598,1538,1371,1151,1138$, 1081, 877, 814, 772; ${ }^{1} \mathrm{H}$ NMR (400 $\left.\mathrm{MHz}, \mathrm{CDCl}_{3}\right): \delta(\mathrm{ppm})=$ 7.29-7.22 (m, 2H, $\mathrm{H}_{\mathrm{ar}}$ ), 7.09-7.08 (m, 1H, Har), 6.99 (dtd, ${ }^{3} J=$ $7.1 \mathrm{~Hz},{ }^{4} J=1.8 \mathrm{~Hz}, 0.8 \mathrm{~Hz}, 1 \mathrm{H}, \mathrm{H}_{\mathrm{ar}}$ ), $4.86\left(\mathrm{~d},{ }^{3} J=10.0 \mathrm{~Hz}, 1 \mathrm{H}\right.$, $\mathrm{H}-4^{\prime}$ ), 3.93 (d, $\left.{ }^{3} J=10.0 \mathrm{~Hz}, 1 \mathrm{H}, \mathrm{H}-1^{\prime}\right), 1.24$ (s, 3H, $\left.\mathrm{CH}_{3}-3^{\prime}\right), 1.18$ (s, 3H, $\left.\mathrm{CH}_{3}-2^{\prime}\right), 1.14\left(\mathrm{~s}, 3 \mathrm{H}, \mathrm{CH}_{3}-3^{\prime}\right), 0.72\left(\mathrm{~s}, 3 \mathrm{H}, \mathrm{CH}_{3}-2^{\prime}\right)$; ${ }^{13} \mathrm{C}$ NMR $\left(101 \mathrm{MHz}, \mathrm{CDCl}_{3}\right): \delta(\mathrm{ppm})=138.6(\mathrm{~s}, \mathrm{C}-3), 134.7(\mathrm{~s}$, C-1), $129.9\left(\mathrm{~d}, \mathrm{C}_{\mathrm{ar}} \mathrm{H}\right), 127.3\left(\mathrm{~d}, \mathrm{C}_{\mathrm{ar}} \mathrm{H}\right), 127.2\left(\mathrm{~d}, \mathrm{C}_{\mathrm{ar}} \mathrm{H}\right), 125.2$ $\left(\mathrm{d}, \mathrm{C}_{\mathrm{ar}} \mathrm{H}\right), 84.7\left(\mathrm{~d}, \mathrm{C}-4^{\prime}\right), 49.2\left(\mathrm{~d}, \mathrm{C}-1^{\prime}\right), 45.0$ (s, C-3'), 39.5 (s, C-2'), 24.3 [q, $\left(\mathrm{C}-2^{\prime}\right) \mathrm{CH}_{3}$ ] 22.7 [q, $\left(\mathrm{C}-3^{\prime}\right) \mathrm{CH}_{3}$ ], 21.5 [q, (C-2') $\mathrm{CH}_{3}$ ], 19.4 [q, $\left(\mathrm{C}-3^{\prime}\right) \mathrm{CH}_{3}$ ]; $\mathrm{MS}(\mathrm{EI}): m / z(\%)=221$ (32) $[\mathrm{M}-$ $\left.\mathrm{NO}_{2}\right]^{+}, 179$ (100) $\left[\mathrm{M}-\mathrm{NO}_{2}-\mathrm{C}_{3} \mathrm{H}_{6}\right]^{+}, 125(32)\left[\mathrm{C}_{7} \mathrm{H}_{6} \mathrm{Cl}\right]^{+} ; \mathrm{HRMS}$ (ESI): calcd for $\mathrm{C}_{14} \mathrm{H}_{19} \mathrm{ClNO}_{2}{ }^{+}[\mathrm{M}+\mathrm{H}]^{+}$: 268.1099; found: 268.1098 .

3-(2',2',3',3'-Tetramethyl-4'-nitrocyclobutyl)-benzonitrile (2k). The reaction was performed in analogy to the representative procedure for conditions A (see above) with nitroethene $\mathbf{1 k}$ (34.8 mg, $200 \mu \mathrm{mol}, 1.00$ equiv.) and 2,3-dimethyl-2-butene (237 $\mu \mathrm{L}, 168 \mathrm{mg}, 2.00 \mathrm{mmol}, 10.0$ equiv.) in dichloromethane $(10 \mathrm{~mL}, c=20 \mathrm{~mm})(t=7 \mathrm{~h})$. Purification by column chromatography $\left(\mathrm{P} / \mathrm{Et}_{2} \mathrm{O}=19 / 1\right)$ yielded $2 \mathrm{k}(18.2 \mathrm{mg}, 70.5 \mu \mathrm{mol}, 35 \%)$ and the by-product $6(2.32 \mathrm{mg}, 8.99 \mu \mathrm{mol}, 11 \%)$ as a mixture 
(colourless liquid). 2k: $R_{\mathrm{f}}=0.53\left(\mathrm{P} / \mathrm{Et}_{2} \mathrm{O}=9 / 1\right) ; \mathrm{IR}: \tilde{\nu}\left(\mathrm{cm}^{-1}\right)=$ 3079, 2960，2231，1533，1372，1151，1136，793; ${ }^{1} \mathrm{H}$ NMR $\left(400 \mathrm{MHz}, \mathrm{CDCl}_{3}\right): \delta(\mathrm{ppm})=7.59-7.55\left(\mathrm{~m}, 1 \mathrm{H}, \mathrm{H}_{\mathrm{ar}}\right), 7.46(\mathrm{td}$, $\left.{ }^{3} J=7.7 \mathrm{~Hz},{ }^{4} J=0.6 \mathrm{~Hz}, 1 \mathrm{H}, \mathrm{H}_{\mathrm{ar}}\right), 7.41-7.39\left(\mathrm{~m}, 1 \mathrm{H}, \mathrm{H}_{\mathrm{ar}}\right)$, 7.36-7.33 (m, 1H, $\left.\mathrm{H}_{\mathrm{ar}}\right), 4.87$ (d, $\left.{ }^{3} J=10.0 \mathrm{~Hz}, 1 \mathrm{H}, \mathrm{H}-4^{\prime}\right), 3.97(\mathrm{~d}$, $\left.{ }^{3} J=10.0 \mathrm{~Hz}, 1 \mathrm{H}, \mathrm{H}-1^{\prime}\right), 1.25$ (s, 3H, $\left.\mathrm{CH}_{3}-3^{\prime}\right), 1.20(\mathrm{~s}, 3 \mathrm{H}$, $\left.\mathrm{CH}_{3}-2^{\prime}\right), 1.16\left(\mathrm{~s}, 3 \mathrm{H}, \mathrm{CH}_{3}-3^{\prime}\right), 0.71\left(\mathrm{~s}, 3 \mathrm{H}, \mathrm{CH}_{3}-2^{\prime}\right) ;{ }^{13} \mathrm{C} \mathrm{NMR}$ $\left(101 \mathrm{MHz}, \mathrm{CDCl}_{3}\right): \delta(\mathrm{ppm})=138.2(\mathrm{~s}, \mathrm{C}-3), 131.5\left(\mathrm{~d}, \mathrm{C}_{\mathrm{ar}} \mathrm{H}\right)$, $130.9\left(\mathrm{~d}, \mathrm{C}_{\mathrm{ar}} \mathrm{H}\right), 130.6\left(\mathrm{~d}, \mathrm{C}_{\mathrm{ar}} \mathrm{H}\right), 129.6\left(\mathrm{~d}, \mathrm{C}_{\mathrm{ar}} \mathrm{H}\right), 118.7(\mathrm{~s}$, $\mathrm{C}_{\mathrm{ar}} \mathrm{CN}$ ), 113.0 (s, C-1), 84.4 (d, C-4'), 49.1 (d, C-1'), 45.2 (s, C-3'), 39.6 (s, C-2'), 24.3 [q, (C-2') $\left.\mathrm{CH}_{3}\right], 22.7$ [q, $\left.\left(\mathrm{C}^{\prime} 3^{\prime}\right) \mathrm{CH}_{3}\right], 21.5$ [q, $\left.\left(\mathrm{C}-2^{\prime}\right) \mathrm{CH}_{3}\right], 19.4$ [q, $\left.\left(\mathrm{C}-3^{\prime}\right) \mathrm{CH}_{3}\right] ; \mathrm{MS}(\mathrm{EI}): m / z(\%)=212(32)$ $\left[\mathrm{M}-\mathrm{NO}_{2}\right]^{+}, 170(100)\left[\mathrm{M}-\mathrm{NO}_{2}-\mathrm{C}_{3} \mathrm{H}_{6}\right]^{+}, 116(20)\left[\mathrm{C}_{8} \mathrm{H}_{6} \mathrm{~N}\right]^{+}$; HRMS (ESI): calcd for $\mathrm{C}_{15} \mathrm{H}_{19} \mathrm{~N}_{2} \mathrm{O}_{2}{ }^{+}[\mathrm{M}+\mathrm{H}]^{+}:$259.1440; found: 259.1442. 6: $R_{\mathrm{f}}=0.53\left(\mathrm{P} / \mathrm{Et}_{2} \mathrm{O}=9 / 1\right)$; IR: $\tilde{\nu}\left(\mathrm{cm}^{-1}\right)=2923,2231$, 1549, 1365, 1148, 905, 798, 691; ${ }^{1} \mathrm{H}$ NMR $\left(500 \mathrm{MHz}, \mathrm{CDCl}_{3}\right)$ : $\delta(\mathrm{ppm})=7.54\left(\mathrm{dd},{ }^{3} J=7.6 \mathrm{~Hz},{ }^{4} J=1.6 \mathrm{~Hz}, 1 \mathrm{H}, \mathrm{H}-4\right)^{*}$, 7.41-7.38 (m, 2H, H-2, H-5), $7.35\left(\mathrm{~d},{ }^{3} J=8.0 \mathrm{~Hz}, 1 \mathrm{H}, \mathrm{H}-6\right)^{*}$, $5.04\left(\mathrm{~s}, 1 \mathrm{H}, \mathrm{C} H \mathrm{H}-5^{\prime}\right), 5.00\left(\mathrm{~s}, 1 \mathrm{H}, \mathrm{CH} H-5^{\prime}\right), 4.76\left(\mathrm{dd},{ }^{3} J=\right.$ $\left.12.0 \mathrm{~Hz}, 2.2 \mathrm{~Hz}, 1 \mathrm{H}, \mathrm{H}-2^{\prime}\right), 3.32\left(\mathrm{dd},{ }^{2} J=15.0,{ }^{3} J=11.9 \mathrm{~Hz}, 1 \mathrm{H}\right.$, $\left.\mathrm{C} H \mathrm{H}-1^{\prime}\right), 2.94\left(\mathrm{dd},{ }^{2} J=15.0,{ }^{3} J=2.2 \mathrm{~Hz}, 1 \mathrm{H}, \mathrm{CH} H-1^{\prime}\right), 1.87(\mathrm{~d}$, $\left.{ }^{4} J=1.4 \mathrm{~Hz}, 3 \mathrm{H}, \mathrm{CH}_{3}-4^{\prime}\right), 1.29\left(\mathrm{~s}, 3 \mathrm{H}, \mathrm{CH}_{3}-3^{\prime}\right), 1.23(\mathrm{~s}, 3 \mathrm{H}$, $\left.\mathrm{CH}_{3}-3^{\prime}\right)$ (* the assignments are interconvertible); ${ }^{13} \mathrm{C}$ NMR $\left(126 \mathrm{MHz}, \mathrm{CDCl}_{3}\right): \delta(\mathrm{ppm})=147.3\left(\mathrm{~s}, \mathrm{C}-4^{\prime}\right), 138.3(\mathrm{~s}, \mathrm{C}-3)$, 133.3 (d, C-6)* 132.4 (d, C-2), 131.2 (d, C-4)* 129.9 (d, C-5), 118.6 (s, CN), 114.5 (t, C-5'), 113.2 (s, C-1), 96.0 (d, C-2'), 43.0 (s, C-3'), 34.4 (t, C-1'), 24.7 [q, $\left.\left(\mathrm{C}-3^{\prime}\right) \mathrm{CH}_{3}\right], 21.6$ [q, $\left(\mathrm{C}-3^{\prime}\right) \mathrm{CH}_{3}$ ], 19.6 [q, $\left.\left(\mathrm{C}-4^{\prime}\right) \mathrm{CH}_{3}\right]$ ( ${ }^{*}$ the assignments are interconvertible); MS (EI): $m / z(\%)=196(15)\left[\mathrm{C}_{14} \mathrm{H}_{14} \mathrm{~N}\right]^{+}, 170(58)\left[\mathrm{C}_{12} \mathrm{H}_{12} \mathrm{~N}\right]^{+}, 116$ (100) $\left[\mathrm{C}_{8} \mathrm{H}_{6} \mathrm{~N}\right]^{+}$; HRMS (ESI): calcd for $\mathrm{C}_{15} \mathrm{H}_{19} \mathrm{~N}_{2} \mathrm{O}_{2}{ }^{+}[\mathrm{M}+\mathrm{H}]^{+}$: 259.1441; found: 259.1439 .

1-Chloro-2-(2',2',3',3'-tetramethyl-4'-nitrocyclobutyl)-benzene (21). The reaction was performed in analogy to the representative procedure for conditions $\mathrm{C}$ (see above) with nitroethene $\mathbf{1 l}$ (18.4 mg, $100 \mu \mathrm{mol}, 1.00$ equiv.) and 2,3-dimethyl-2-butene (119 $\mu \mathrm{L}, 84.2 \mathrm{mg}, 1.00 \mathrm{mmol}, 10.0$ equiv.) in dichloromethane $(5 \mathrm{~mL}, c=20 \mathrm{~mm})(t=7 \mathrm{~h})$. Purification by column chromatography $\left(\mathrm{P} / \mathrm{Et}_{2} \mathrm{O}=19 / 1\right)$ yielded $2 \mathbf{l}(7.46 \mathrm{mg}, 27.9 \mu \mathrm{mol}, 28 \%)$ as a colourless oil. Starting material was recovered as cis-isomer cis-11 (7.94 mg, $43.2 \mu \mathrm{mol}, 43 \%) . R_{\mathrm{f}}=0.54\left(\mathrm{P} / \mathrm{Et}_{2} \mathrm{O}=9 / 1\right)$; IR: $\tilde{\nu}\left(\mathrm{cm}^{-1}\right)=3061,2973,2960,1541,1372,1153,1135,1033,876$, 807,$754 ;{ }^{1} \mathrm{H}$ NMR $\left(400 \mathrm{MHz}, \mathrm{CDCl}_{3}\right): \delta(\mathrm{ppm})=7.41\left(\mathrm{dd},{ }^{3} J=\right.$ $\left.7.6 \mathrm{~Hz},{ }^{4} J=1.6 \mathrm{~Hz}, 1 \mathrm{H}, \mathrm{H}-6\right), 7.28-7.18$ (m, 2H, H-5, H-4), 7.16 $\left(\mathrm{dd},{ }^{3} J=7.5 \mathrm{~Hz},{ }^{4} J=1.9 \mathrm{~Hz}, 1 \mathrm{H}, \mathrm{H}-3\right), 4.99\left(\mathrm{~d},{ }^{3} J=10.2 \mathrm{~Hz}, 1 \mathrm{H}\right.$, $\left.\mathrm{H}-4^{\prime}\right), 4.43\left(\mathrm{~d},{ }^{3} \mathrm{~J}=10.2 \mathrm{~Hz}, 1 \mathrm{H}, \mathrm{H}-1^{\prime}\right), 1.26$ (s, 3H, $\left.\mathrm{CH}_{3}-2^{\prime}\right), 1.24$ (s, 3H, $\left.\mathrm{CH}_{3}-3^{\prime}\right), 1.19$ (s, 3H, $\left.\mathrm{CH}_{3}-3^{\prime}\right), 0.73$ (s, 3H, $\mathrm{CH}_{3}-2^{\prime}$ ); ${ }^{13} \mathrm{C}$ NMR $\left(101 \mathrm{MHz}, \mathrm{CDCl}_{3}\right): \delta(\mathrm{ppm})=134.7(\mathrm{~s}, \mathrm{C}-2), 134.1$ (s, C-1), 130.3 (d, C-6), 128.4 (d, C-5/C-4)* 128.1 (d, C-3), 126.8 (d, C-5/C-4)*, 84.4 (d, C-4'), 46.8 (d, C-1'), 44.5 (s, C-2'), 40.5 (s, C-3'), 24.9 [q, $\left(\mathrm{C}-3^{\prime}\right) \mathrm{CH}_{3}$ ], 22.7 [q, $\left(\mathrm{C}-2^{\prime}\right) \mathrm{CH}_{3}$ ] 21.8 [q, $\left(\mathrm{C}-2^{\prime}\right)$ $\left.\mathrm{CH}_{3}\right], 19.4$ [q, $\left.\left(\mathrm{C}-3^{\prime}\right) \mathrm{CH}_{3}\right]\left({ }^{*}\right.$ the assignments are interconvertible); $\mathrm{MS}(\mathrm{EI}): \mathrm{m} / \mathrm{z}(\%)=221(32)\left[\mathrm{M}-\mathrm{NO}_{2}\right]^{+}, 179(100)$ $\left[\mathrm{M}-\mathrm{NO}_{2}-\mathrm{C}_{3} \mathrm{H}_{6}\right]^{+}, 125(28)\left[\mathrm{C}_{7} \mathrm{H}_{6} \mathrm{Cl}\right]^{+}$; HRMS (ESI): calcd for $\mathrm{C}_{14} \mathrm{H}_{19} \mathrm{ClNO}_{2}{ }^{+}[\mathrm{M}+\mathrm{H}]^{+}$: 268.1099; found: 268.1094.

2-(2',2',3',3'-Tetramethyl-4'-nitrocyclobutyl)-pyridine

(2m). The reaction was performed in analogy to the representative procedure for conditions $\mathrm{C}$ (see above) with nitroethene $1 \mathrm{~m}$ (15.0 mg, $100 \mu \mathrm{mol}, 1.00$ equiv.) and 2,3-dimethyl-2-butene (119 $\mu \mathrm{L}, 84.2 \mathrm{mg}, 1.00 \mathrm{mmol}, 10.0$ equiv.) in dichloromethane $(5 \mathrm{~mL}, c=20 \mathrm{~mm})(t=24 \mathrm{~h})$. Purification by column chromatography $\left(\mathrm{CH}_{2} \mathrm{Cl}_{2} / \mathrm{MeOH}=40 / 1\right)$ yielded $2 \mathrm{~m}(15.5 \mathrm{mg}, 66.2 \mu \mathrm{mol}$, $66 \%)$ as a yellow coloured oil. $R_{\mathrm{f}}=0.25\left(\mathrm{CH}_{2} \mathrm{Cl}_{2} / \mathrm{MeOH}=19 / 1\right)$; IR: $\tilde{\nu}\left(\mathrm{cm}^{-1}\right)=3445,3008,2930,1597,1448,1386,1225,1052$, 1025, 992, 760; ${ }^{1} \mathrm{H}$ NMR $\left(500 \mathrm{MHz}, \mathrm{CDCl}_{3}\right): \delta(\mathrm{ppm}) 8.59-8.37$ (m, 2H, H-5, H-6), 7.43 (dt, ${ }^{3} J=7.9 \mathrm{~Hz},{ }^{4} J=2.0 \mathrm{~Hz}, 1 \mathrm{H}, \mathrm{H}-3$ ), 7.31-7.27 (m, 1H, H-4), 4.90 (d, $\left.{ }^{3} J=10.0 \mathrm{~Hz}, 1 \mathrm{H}, \mathrm{H}-4^{\prime}\right), 3.96$ (d, $\left.{ }^{3} J=10.0 \mathrm{~Hz}, 1 \mathrm{H}, \mathrm{H}-1^{\prime}\right), 1.25$ (s, 3H, $\left.\mathrm{CH}_{3}-2^{\prime}\right), 1.19$ (s, 3H, $\left.\mathrm{CH}_{3}-3^{\prime}\right), 1.16\left(\mathrm{~s}, 3 \mathrm{H}, \mathrm{CH}_{3}-2^{\prime}\right), 0.73\left(\mathrm{~s}, 3 \mathrm{H}, \mathrm{CH}_{3}-3^{\prime}\right) ;{ }^{13} \mathrm{C}$ NMR $\left(126 \mathrm{MHz}, \mathrm{CDCl}_{3}\right): \delta(\mathrm{ppm})=148.7(\mathrm{~d}, \mathrm{C}-5 / \mathrm{C}-6)^{*}, 148.6(\mathrm{~d}, \mathrm{C}-5 /$ C-6)* 134.5 (d, C-3), 131.9 (s, C-2), 123.4 (d, C-4), 84.2 (d, C-4'), 47.4 (d, C-1'), 45.3 (s, C-3'), 39.4 (s, C-2'), 24.2 [q, $\left(\mathrm{C}-2^{\prime}\right) \mathrm{CH}_{3}$ ], $22.7\left[\mathrm{q},\left(\mathrm{C}-3^{\prime}\right) \mathrm{CH}_{3}\right], 21.6\left[\mathrm{q},\left(\mathrm{C}-2^{\prime}\right) \mathrm{CH}_{3}\right], 19.3\left[\mathrm{q},\left(\mathrm{C}-3^{\prime}\right) \mathrm{CH}_{3}\right]\left({ }^{*}\right.$ the assignments are interconvertible); MS (EI): $m / z(\%)=188(100)$ $\left[\mathrm{M}-\mathrm{NO}_{2}\right]^{+}, 146(72)\left[\mathrm{M}-\mathrm{NO}_{2}-\mathrm{C}_{3} \mathrm{H}_{6}\right]^{+}, 132(40)\left[\mathrm{C}_{9} \mathrm{H}_{10} \mathrm{~N}\right]^{+}$; HRMS (ESI): calcd for $\mathrm{C}_{13} \mathrm{H}_{19} \mathrm{~N}_{2} \mathrm{O}_{2}{ }^{+}[\mathrm{M}+\mathrm{H}]^{+}$: 235.1441; found: 235.1441.

2-(2', 2',3',3'-Tetramethyl-4'-nitrocyclobutyl)-naphthalene (2n). The reaction was performed in analogy to the representative procedure for conditions $\mathrm{C}$ (see above) with nitroethene $\mathbf{1 n}$ (19.9 mg, $100 \mu \mathrm{mol}, 1.00$ equiv.) and 2,3-dimethyl-2-butene (119 $\mu \mathrm{L}, 84.2 \mathrm{mg}, 1.00 \mathrm{mmol}, 10.0$ equiv.) in dichloromethane $(5 \mathrm{~mL}, c=20 \mathrm{~mm})(t=3 \mathrm{~h})$. Purification by column chromatography $\left(\mathrm{P} / \mathrm{Et}_{2} \mathrm{O}=19 / 1\right)$ yielded $2 \mathrm{n}(22.4 \mathrm{mg}, 79.1 \mu \mathrm{mol}, 79 \%)$ as a yellow coloured oil. $R_{\mathrm{f}}=0.56\left(\mathrm{P} / \mathrm{Et}_{2} \mathrm{O}=9 / 1\right)$; IR: $\tilde{\nu}\left(\mathrm{cm}^{-1}\right)=$ 2954, 1537, 1459, 1367, 1139, 858, 810, 755; ${ }^{1} \mathrm{H}$ NMR $\left(500 \mathrm{MHz}, \mathrm{CDCl}_{3}\right): \delta(\mathrm{ppm})=7.85-7.79\left(\mathrm{~m}, 3 \mathrm{H}, \mathrm{H}_{\mathrm{ar}}\right), 7.54-7.52$ (br. s., $\left.1 \mathrm{H}, \mathrm{H}_{\mathrm{ar}}\right), 7.51-7.43\left(\mathrm{~m}, 2 \mathrm{H}, \mathrm{H}_{\mathrm{ar}}\right), 7.26-7.23\left(\mathrm{~m}, 1 \mathrm{H}, \mathrm{H}_{\mathrm{ar}}\right.$ ), $5.06\left(\mathrm{~d},{ }^{3} J=10.1 \mathrm{~Hz}, 1 \mathrm{H}, \mathrm{H}-4^{\prime}\right), 4.14\left(\mathrm{~d},{ }^{3} J=10.1 \mathrm{~Hz}, 1 \mathrm{H}, \mathrm{H}-1^{\prime}\right)$, $1.28\left(\mathrm{~s}, 3 \mathrm{H}, \mathrm{CH}_{3}-2^{\prime}\right), 1.26$ (s, 3H, $\left.\mathrm{CH}_{3}-3^{\prime}\right), 1.20\left(\mathrm{~s}, 3 \mathrm{H}, \mathrm{CH}_{3}-2^{\prime}\right)$, $0.73\left(\mathrm{~s}, 3 \mathrm{H}, \mathrm{CH}_{3}-3^{\prime}\right) ;{ }^{13} \mathrm{C}$ NMR $\left(101 \mathrm{MHz}, \mathrm{CDCl}_{3}\right): \delta(\mathrm{ppm})=$ 134.2 (s, C-2), 133.5 (s, C-5/C-10)*, 132.6 (s, C-5/C-10)*, 128.4 $\left(d, \mathrm{C}_{a r} \mathrm{H}\right), 127.8\left(\mathrm{~d}, \mathrm{C}_{\mathrm{ar}} \mathrm{H}\right), 126.5\left(\mathrm{~d}, \mathrm{C}_{\mathrm{ar}} \mathrm{H}\right), 125.9\left(\mathrm{~d}, \mathrm{C}_{\mathrm{ar}} \mathrm{H}\right)$, $125.7\left(\mathrm{~d}, \mathrm{C}_{\mathrm{ar}} \mathrm{H}\right), 125.1\left(\mathrm{~d}, \mathrm{C}_{\mathrm{ar}} \mathrm{H}\right), 85.0\left(\mathrm{~d}, \mathrm{C}-4^{\prime}\right), 49.6$ (d, C-1'), 45.1 (s, C-3'), 39.5 (s, C-2'), 24.4 [q, (C-2') $\mathrm{CH}_{3}$ ], 22.8 [q, (C-3') $\left.\mathrm{CH}_{3}\right], 21.6\left[\mathrm{q},\left(\mathrm{C}-2^{\prime}\right) \mathrm{CH}_{3}\right], 19.5\left[\mathrm{q},\left(\mathrm{C}-3^{\prime}\right) \mathrm{CH}_{3}\right]\left({ }^{*}\right.$ the assignments are interconvertible); MS (EI): $\mathrm{m} / z(\%)=237(28)\left[\mathrm{M}-\mathrm{NO}_{2}\right]^{+}$, 181 (100) $\left[\mathrm{M}-\mathrm{NO}_{2}-\mathrm{C}_{4} \mathrm{H}_{8}\right]^{+}, 127(10)\left[\mathrm{C}_{10} \mathrm{H}_{7}\right]^{+}$; HRMS (ESI): calcd for $\mathrm{C}_{18} \mathrm{H}_{22} \mathrm{NO}_{2}^{+}[\mathrm{M}+\mathrm{H}]^{+}: 284.1645$; found: 284.1646 .

2-Nitro-1-phenylspiro[3.5] nonane (3a). The reaction was performed in analogy to the representative procedure for conditions C (see above) with nitroethene 1a $(14.9 \mathrm{mg}, 100 \mu \mathrm{mol}$, 1.00 equiv.) and methylenecyclohexane $(136 \mu \mathrm{L}, 96.1 \mathrm{mg}$, $1.00 \mathrm{mmol}, 10.0$ equiv.) in dichloromethane ( $5 \mathrm{~mL}, c=20 \mathrm{~mm})$ $(t=14 \mathrm{~h})$. The crude product was purified by column chromatography $\left(\mathrm{P} / \mathrm{Et}_{2} \mathrm{O}=20 / 1\right)$ to yield $3 \mathrm{a}(15.0 \mathrm{mg}, 61.9 \mu \mathrm{mol}, 61 \%)$ as a colourless oil. Starting material 1a was recovered as a mixture of isomers $(6.64 \mathrm{mg}, 42.9 \mu \mathrm{mol}, 43 \%$, cis/trans $=$ $12: 88)$. The analytical data obtained matched those reported in the literature. ${ }^{13}$

6-Nitro-7-phenyl-2-oxabicyclo[3.2.0]heptane (3b). The reaction was performed in analogy to the representative procedure for conditions B (see above) with nitroethene 1a (14.9 mg, 
$100 \mu \mathrm{mol}, 1.00$ equiv.) and 2,3-dihydrofuran (75.5 $\mu \mathrm{L}, 70.0 \mathrm{mg}$, $1.00 \mathrm{mmol}, 10.0$ equiv.) in dichloromethane ( $5 \mathrm{~mL}, c=20 \mathrm{~mm}$ ) $(t=6 \mathrm{~h})$. The crude product was purified by column chromatography $\left(\mathrm{P} / \mathrm{Et}_{2} \mathrm{O}=9 / 1 \rightarrow 4 / 1\right)$ to yield $3 \mathbf{b}(8.10 \mathrm{mg}, 36.9 \mu \mathrm{mol}$, $37 \%$ ) as a yellow coloured oil. Starting material was recovered as cis-isomer cis-1a (2.00 mg, $13.4 \mu \mathrm{mol}, 13 \%)$. The analytical data obtained matched those reported in the literature. ${ }^{13}$

(2',2'-Diethyl-4'-nitrocyclobutyl)-benzene (3c). The reaction was performed in analogy to the representative procedure for conditions A (see above) with nitroethene 1a $(29.8 \mathrm{mg}$, $200 \mu \mathrm{mol}, 1.00$ equiv.) and 1,1-diethylethylene $(244 \mu \mathrm{L}$, $168 \mathrm{mg}, 2.00 \mathrm{mmol}, 10.0$ equiv.) in dichloromethane $(10 \mathrm{~mL}$, $c=20 \mathrm{~mm})(t=24 \mathrm{~h})$. The crude product was purified by column chromatography $\left(\mathrm{P} / \mathrm{Et}_{2} \mathrm{O}=30 / 1\right)$ to yield $3 \mathrm{c}(20.7 \mathrm{mg}$, $88.7 \mathrm{mmol}, 44 \%$ ) as a yellow coloured oil. Starting material 1a was recovered as a mixture of isomers $(4.70 \mathrm{mg}, 31.5 \mu \mathrm{mol}$, $16 \%$, cis/trans $=44: 56) . R_{\mathrm{f}}=0.45\left(\mathrm{P} / \mathrm{Et}_{2} \mathrm{O}=19 / 1\right) ;$ IR: $\tilde{\nu}\left(\mathrm{cm}^{-1}\right)=$ 3063, 3030, 2965, 1542, 1455, 1366, 784; ${ }^{1} \mathrm{H}$ NMR $(500 \mathrm{MHz}$, $\left.\mathrm{CDCl}_{3}\right): \delta(\mathrm{ppm})=7.34\left(\mathrm{t},{ }^{3} J=7.5 \mathrm{~Hz}, 2 \mathrm{H}\right.$, meta $\left.-\mathrm{H}_{\mathrm{ar}}\right), 7.29-7.24$ $\left(\mathrm{m}, 1 \mathrm{H}\right.$, para $\left.-\mathrm{H}_{\mathrm{ar}}\right), 7.23-7.20\left(\mathrm{~m}, 2 \mathrm{H}\right.$, ortho- $\left.\mathrm{H}_{\mathrm{ar}}\right), 5.27$ (virt. $\mathrm{q}$, $\left.{ }^{3} J \cong{ }^{3} J=8.7 \mathrm{~Hz}, 1 \mathrm{H}, \mathrm{H}-4^{\prime}\right), 3.98\left(\mathrm{~d},{ }^{3} J=9.1 \mathrm{~Hz}, 1 \mathrm{H}, \mathrm{H}-1^{\prime}\right), 2.40$ $\left(\mathrm{dd},{ }^{2} J=11.9 \mathrm{~Hz},{ }^{3} J=8.5 \mathrm{~Hz}, 1 \mathrm{H}, \mathrm{CHH}-3\right), 2.30\left(\mathrm{dd},{ }^{2} J=\right.$ $\left.11.9 \mathrm{~Hz},{ }^{3} \mathrm{~J}=8.6 \mathrm{~Hz}, 1 \mathrm{H}, \mathrm{CH} H-3\right), 1.76\left(\mathrm{dq},{ }^{2} \mathrm{~J}=14.8 \mathrm{~Hz},{ }^{3} \mathrm{~J}=\right.$ $\left.7.5 \mathrm{~Hz}, 1 \mathrm{H}, \mathrm{CH} \mathrm{HCH}_{3}\right), 1.64\left(\mathrm{dq},{ }^{2} J=14.8 \mathrm{~Hz},{ }^{3} J=7.5 \mathrm{~Hz}, 1 \mathrm{H}\right.$, $\left.\mathrm{CH} \mathrm{CH}_{3}\right), 1.30-1.19\left(\mathrm{~m}, 2 \mathrm{H}, \mathrm{CH}_{2} \mathrm{CH}_{3}\right), 0.96\left(\mathrm{t},{ }^{3} J=7.5 \mathrm{~Hz}, 3 \mathrm{H}\right.$, $\left.\mathrm{CH}_{2} \mathrm{CH}_{3}\right), 0.60\left(\mathrm{t},{ }^{3} \mathrm{~J}=7.4 \mathrm{~Hz}, 3 \mathrm{H}, \mathrm{CH}_{2} \mathrm{CH}_{3}\right) ;{ }^{13} \mathrm{C} \quad \mathrm{NMR}$ $\left(126 \mathrm{MHz}, \mathrm{CDCl}_{3}\right): \delta(\mathrm{ppm})=136.6\left(\mathrm{~s}, \mathrm{C}_{\mathrm{ar}}\right), 128.6(\mathrm{~d}, 2 \mathrm{C}$, meta$\left.\mathrm{C}_{\mathrm{ar}} \mathrm{H}\right), 127.5\left(\mathrm{~d}, 2 \mathrm{C}\right.$, ortho $\left.-\mathrm{C}_{\mathrm{ar}} \mathrm{H}\right), 127.2\left(\mathrm{~d}\right.$, para $\left.-\mathrm{C}_{\mathrm{ar}} \mathrm{H}\right), 76.6$ (d, C-4'), 53.8 (d, C-1'), 41.8 (s, C-2'), 33.7 (t, C-3'), 31.7 (t, $\left.\mathrm{CH}_{2} \mathrm{CH}_{3}\right), 26.4$ (t, $\left.\mathrm{CH}_{2} \mathrm{CH}_{3}\right), 8.62\left(\mathrm{q}, \mathrm{CH}_{2} \mathrm{CH}_{3}\right), \quad 7.98$ (q, $\mathrm{CH}_{2} \mathrm{CH}_{3}$ ); $\mathrm{MS}(\mathrm{EI}): \mathrm{m} / z(\%)=187$ (4) $\left[\mathrm{M}-\mathrm{NO}_{2}\right]^{+}, 157$ (12) $\left[\mathrm{M}-\mathrm{NO}_{2}-\mathrm{C}_{2} \mathrm{H}_{6}\right]^{+}, 117$ (100) $\left[\mathrm{C}_{9} \mathrm{H}_{9}\right]^{+}$; HRMS (ESI): calcd for $\mathrm{C}_{14} \mathrm{H}_{20} \mathrm{NO}_{2}^{+}[\mathrm{M}+\mathrm{H}]^{+}:$234.1488; found: 234.1489.

1-Nitro-2-phenyl-1,2,2a,7b-tetrahydrocyclobuta $[b]$ benzofuran (3d). The reaction was performed in analogy to the representative procedure for conditions B (see above) with nitroethene 1a (14.9 mg, $100 \mu \mathrm{mol}, 1.00$ equiv.) and benzofuran $(108 \mu \mathrm{L}$, $118 \mathrm{mg}, 1.00 \mathrm{mmol}, 10.0$ equiv. $)$ in dichloromethane $(5 \mathrm{~mL}, c=$ $20 \mathrm{~mm})(t=36 \mathrm{~h})$. Purification by column chromatography $(\mathrm{P} /$ $\left.\mathrm{Et}_{2} \mathrm{O}=19 / 1\right)$ yielded $3 \mathbf{d}(12.8 \mathrm{mg}, 47.9 \mu \mathrm{mol}, 48 \%)$ as a yellow coloured oil. Starting material was recovered as cis-isomer cis1a $(5.40 \mathrm{mg}, 36.2 \mu \mathrm{mol}, 36 \%) . R_{\mathrm{f}}=0.53\left(\mathrm{P} / \mathrm{Et}_{2} \mathrm{O}=9 / 1\right)$; IR: $\tilde{\nu}\left(\mathrm{cm}^{-1}\right)=3062$, 3032, 2923, 1543, 1474, 1368, 1218, 1095, 1051, 1019, 814; ${ }^{1} \mathrm{H}$ NMR (500 MHz, $\left.\mathrm{C}_{6} \mathrm{D}_{6}\right): \delta(\mathrm{ppm})=6.98-6.96(\mathrm{~m}$ $3 \mathrm{H}$, meta $-\mathrm{H}_{\mathrm{ar}}$, para $\left.-\mathrm{H}_{\mathrm{ar}}\right), 6.84\left(\mathrm{t},{ }^{3} \mathrm{~J}=7.5 \mathrm{~Hz}, 1 \mathrm{H}, \mathrm{H}-5\right), 6.79(\mathrm{~d}$, $\left.{ }^{3} J=7.5 \mathrm{~Hz}, 1 \mathrm{H}, \mathrm{H}-4\right), 6.61-6.56\left(\mathrm{~m}, 2 \mathrm{H}\right.$, ortho- $\left.\mathrm{H}_{\mathrm{ar}}\right), 6.45\left(\mathrm{t},{ }^{3} J=\right.$ $7.5 \mathrm{~Hz}, 1 \mathrm{H}, \mathrm{H}-6), 6.29$ (d, ${ }^{3} J=7.5 \mathrm{~Hz}, 1 \mathrm{H}, \mathrm{H}-7$ ), 5.13 (dd, ${ }^{3} J=$ $7.4 \mathrm{~Hz}, 4.2 \mathrm{~Hz}, 1 \mathrm{H}, \mathrm{H}-2 \mathrm{a}$ ), 4.97 (ddd, ${ }^{3} \mathrm{~J}=9.4 \mathrm{~Hz}, 4.2 \mathrm{~Hz},{ }^{4} J=1.5$ $\mathrm{Hz}, 1 \mathrm{H}, \mathrm{H}-1$ ), 3.72 (virt. $\mathrm{t},{ }^{3} J \cong{ }^{3} J=9.3 \mathrm{~Hz}, 1 \mathrm{H}, \mathrm{H}-2$ ), 3.50 (virt. t, $\left.{ }^{3} J \cong{ }^{3} J=8.4 \mathrm{~Hz}, 1 \mathrm{H}, \mathrm{H}-7 \mathrm{~b}\right) ;{ }^{13} \mathrm{C} \mathrm{NMR}\left(101 \mathrm{MHz}, \mathrm{C}_{6} \mathrm{D}_{6}\right): \delta(\mathrm{ppm})$ $=160.9(\mathrm{~s}, \mathrm{C}-3 \mathrm{a}), 135.4\left(\mathrm{~s}, \mathrm{C}-2^{\prime}\right), 129.6$ (d, C-5), 128.6 (d, meta$\mathrm{C}_{\mathrm{ar}} \mathrm{H} /$ para $\left.-\mathrm{C}_{\mathrm{ar}} \mathrm{H}\right)^{*}, 128.3(\mathrm{~d}, \mathrm{C}-7), 127.9$ (d, meta $-\mathrm{C}_{\mathrm{ar}} \mathrm{H} /$ para $\left.-\mathrm{C}_{\mathrm{ar}} \mathrm{H}\right)$ *, 127.6 (d, 2C, ortho- $\left.\mathrm{C}_{\mathrm{ar}} \mathrm{H}\right)^{*}, 124.9$ (s, C-7a), 121.6 (d, C-6), 111.5 (d, C-4), 85.6 (d, C-1), 80.6 (d, C-2a), 45.6 (d, C-2), 44.6 (d, C-7b) (* the exact assignment was not possible due to significant overlap with the solvent $\left.\mathrm{C}_{6} \mathrm{D}_{6}\right)$; $\mathrm{MS}(\mathrm{EI}): \mathrm{m} / z(\%)=221$ (12)
$\left[\mathrm{M}-\mathrm{NO}_{2}\right]^{+}, 118$ (100) $\left[\mathrm{C}_{8} \mathrm{H}_{6} \mathrm{O}\right]^{+}, 90$ (8); HRMS (ESI): calcd for $\mathrm{C}_{16} \mathrm{H}_{14} \mathrm{NO}_{3}^{+}[\mathrm{M}+\mathrm{H}]^{+}$: 268.0968; found: 268.0970.

7-Nitro-8-phenyl-2,5-dioxabicyclo[4.2.0] octane (3e). The reaction was performed in analogy to the representative procedure for conditions A (see above) with nitroethene 1a $29.8 \mathrm{mg}$, $200 \mu \mathrm{mol}, 1.00$ equiv.) and 2,3-dihydro-1,4-dioxin $(159 \mu \mathrm{L}$, $172 \mathrm{mg}, 2.00 \mathrm{mmol}, 10.0$ equiv.) in dichloromethane $(10 \mathrm{~mL}$, $c=20 \mathrm{~mm})(t=14 \mathrm{~h})$. Purification by column chromatography $\left(\mathrm{P} / \mathrm{Et}_{2} \mathrm{O}=9 / 1 \rightarrow 4 / 1\right)$ yielded $3 \mathrm{e}(34.0 \mathrm{mg}, 145 \mu \mathrm{mol}, 72 \%, \mathrm{dr}=$ $52: 19: 29)$ as an orange coloured oil. Starting material 1a was recovered as a mixture of isomers $(4.50 \mathrm{mg}, 30.2 \mu \mathrm{mol}, 15 \%$, cis/trans $=55: 45)$. NMR data are given for the major diastereoisomer depicted in Scheme 2. $R_{\mathrm{f}}=0.06\left(\mathrm{P} / \mathrm{Et}_{2} \mathrm{O}=4 / 1\right)$; IR: $\tilde{\nu}\left(\mathrm{cm}^{-1}\right)=3031,2923,1545,1375,1132,1043,874,751 ;{ }^{1} \mathrm{H}$ NMR $\left(400 \mathrm{MHz}, \mathrm{C}_{6} \mathrm{D}_{6}\right): \delta(\mathrm{ppm})=7.02-6.93\left(\mathrm{~m}, 5 \mathrm{H}, \mathrm{H}_{\mathrm{ar}}\right), 4.26$ (virt. $\mathrm{t}$, ${ }^{3} J \cong{ }^{3} J=7.8 \mathrm{~Hz}, 1 \mathrm{H}, \mathrm{H}-7$ ), 3.81 (virt. $\mathrm{t},{ }^{3} \mathrm{~J} \cong{ }^{3} J=8.6 \mathrm{~Hz}, 1 \mathrm{H}, \mathrm{H}-6$ ), $3.56\left(\mathrm{dd},{ }^{3} \mathrm{~J}=9.8 \mathrm{~Hz}, 7.3 \mathrm{~Hz}, 1 \mathrm{H}, \mathrm{H}-8\right), 3.40-3.28(\mathrm{~m}, 2 \mathrm{H}$, $\mathrm{CHH}-3, \mathrm{CHH}-4), 3.19-3.15$ (m, 2H, CHH-3, CHH-4), 2.99 (d, ${ }^{3} J=$ $9.8 \mathrm{~Hz}, 1 \mathrm{H}, \mathrm{H}-1) ;{ }^{13} \mathrm{C}$ NMR $\left(101 \mathrm{MHz}, \mathrm{C}_{6} \mathrm{D}_{6}\right): \delta(\mathrm{ppm})=136.5$ $\left(\mathrm{s}, \mathrm{C}_{\mathrm{ar}}\right), 129.0\left(\mathrm{~d}, 2 \mathrm{C} \text {, ortho }-\mathrm{C}_{\mathrm{ar}} \mathrm{H}\right)^{*}, 127.9\left(\mathrm{~d}, 2 \mathrm{C}, \text { meta }-\mathrm{C}_{\mathrm{ar}} \mathrm{H}\right)^{*}$, 126.9 (d, para $\left.-\mathrm{C}_{\mathrm{ar}} \mathrm{H}\right), 82.3$ (d, C-7), 77.9 (d, C-6), 75.2 (d, C-1), $68.3(\mathrm{t}, \mathrm{C}-3), 68.1(\mathrm{t}, \mathrm{C}-4), 51.2(\mathrm{~d}, \mathrm{C}-8)$ (* the assignments are interconvertible); MS (EI): $m / z(\%)=235(16)[\mathrm{M}]^{+}, 189(52)[\mathrm{M}$ $\left.-\mathrm{NO}_{2}\right]^{+}, 117$ (72) $\left[\mathrm{C}_{9} \mathrm{H}_{9}\right]^{+}, 91$ (100) $\left[\mathrm{C}_{7} \mathrm{H}_{7}\right]^{+}$; HRMS (ESI): calcd for $\mathrm{C}_{12} \mathrm{H}_{14} \mathrm{NO}_{4}^{+}[\mathrm{M}+\mathrm{H}]^{+}$: 236.0917; found: 236.0918 .

(1'-Cyclopropyl-3'-nitrocyclobutane-1',2'-diyl)-dibenzene (3f). The reaction was performed in analogy to the representative procedure for conditions A (see above) with nitroethene 1a (14.9 mg, $100 \mu \mathrm{mol}, 1.00$ equiv.) and (1-cyclopropylvinyl)benzene (144 mg, $1.00 \mathrm{mmol}, 10.0$ equiv.) in dichloromethane ( $5 \mathrm{~mL}, c=20 \mathrm{~mm})(t=16 \mathrm{~h})$. Purification by column chromatography $\left(\mathrm{P} / \mathrm{Et}_{2} \mathrm{O}=20 / 1\right)$ yielded $3 f(25.6 \mathrm{mg}, 86.6 \mu \mathrm{mol}, 88 \%$, $\mathrm{dr}=67: 33)$ as a pale-yellow coloured oil. NMR data are given for the major diastereoisomer depicted in Scheme 2. $R_{\mathrm{f}}=0.69$ $\left(\mathrm{P} / \mathrm{Et}_{2} \mathrm{O}=9 / 1\right)$; IR: $\tilde{\nu}\left(\mathrm{cm}^{-1}\right)=3028,1542,1496,1368,1028,824$, $770 ;{ }^{1} \mathrm{H}$ NMR $\left(500 \mathrm{MHz}, \mathrm{CDCl}_{3}\right): \delta(\mathrm{ppm})=7.17-7.13(\mathrm{~m}, 6 \mathrm{H}$, $\mathrm{H}_{\mathrm{ar}}$ ), 7.00-6.98 (m, 2H, $\mathrm{H}_{\mathrm{ar}}$ ), 6.83-6.77 (m, 2H, $\mathrm{H}_{\mathrm{ar}}$ ), 5.13 (virt. q, $\left.{ }^{3} J \cong{ }^{3} J=9.0 \mathrm{~Hz}, 1 \mathrm{H}, \mathrm{H}-3^{\prime}\right), 4.07$ (d, $\left.{ }^{3} J=9.5 \mathrm{~Hz}, 1 \mathrm{H}, \mathrm{H}-4^{\prime}\right), 3.01$ $\left(\mathrm{dd},{ }^{2} J=12.3 \mathrm{~Hz},{ }^{3} J=8.1 \mathrm{~Hz}, 1 \mathrm{H}, \mathrm{CHH}-2^{\prime}\right), 2.60\left(\mathrm{dd},{ }^{2} J=12.4\right.$ $\left.\mathrm{Hz},{ }^{3} J=9.2 \mathrm{~Hz}, 1 \mathrm{H}, \mathrm{CH} H-2^{\prime}\right), 1.43\left[\mathrm{tt},{ }^{3} J=8.3 \mathrm{~Hz}, 5.6 \mathrm{~Hz}, 1 \mathrm{H}\right.$, $\left.\mathrm{CH}\left(\mathrm{CH}_{2}\right)_{2}\right], 0.77-0.66\left[\mathrm{~m}, 2 \mathrm{H}, \mathrm{CH}\left(\mathrm{CH}_{2}\right)_{2}\right], 0.57\left[\right.$ virt. tt, ${ }^{2} \mathrm{~J} \cong{ }^{3} \mathrm{~J} \cong$ $\left.{ }^{3} J=8.6 \mathrm{~Hz},{ }^{3} J=5.5 \mathrm{~Hz}, 1 \mathrm{H}, \mathrm{CH}\left(\mathrm{CH}_{2}\right)_{2}\right], 0.44\left[\right.$ virt. dq, ${ }^{2} J=9.0$ $\left.\mathrm{Hz},{ }^{3} J \cong{ }^{3} J=5.5 \mathrm{~Hz}, 1 \mathrm{H}, \mathrm{CH}\left(\mathrm{CH}_{2}\right)_{2}\right] ;{ }^{13} \mathrm{C} \mathrm{NMR}(101 \mathrm{MHz}$, $\left.\mathrm{CDCl}_{3}\right): \delta(\mathrm{ppm})=141.1(\mathrm{~s}, \mathrm{C}-1 \mathrm{a}), 136.2(\mathrm{~s}, \mathrm{C}-4 \mathrm{a}), 128.4(\mathrm{~d}, 2 \mathrm{C}$, $\left.\mathrm{C}_{\mathrm{ar}} \mathrm{H}\right), 128.3$ (d, 2C, $\left.\mathrm{C}_{\mathrm{ar}} \mathrm{H}\right), 128.0\left(\mathrm{~d}, 2 \mathrm{C}, \mathrm{C}_{\mathrm{ar}} \mathrm{H}\right), 127.8\left(\mathrm{~d}, \mathrm{C}_{\mathrm{ar}} \mathrm{H}\right)$, 127.5 (d, $\mathrm{C}_{\mathrm{ar}} \mathrm{H}$ ), 126.8 (d, 2C, $\left.\mathrm{C}_{\mathrm{ar}} \mathrm{H}\right), 76.9$ (d, C-3'), 55.1 (d, C-4'), 47.3 (s, C-1'), 32.1 (t, C-2'), $22.6\left[\mathrm{~d}, \mathrm{CH}\left(\mathrm{CH}_{2}\right)_{2}\right], 3.21[\mathrm{t}, \mathrm{CH}$ $\left.\left(\mathrm{CH}_{2}\right)_{2}\right], 2.11\left[\mathrm{t}, \mathrm{CH}\left(\mathrm{CH}_{2}\right)_{2}\right] ; \mathrm{MS}(\mathrm{EI}): \mathrm{m} / z(\%)=247$ (4) $[\mathrm{M}-$ $\left.\mathrm{NO}_{2}\right]^{+}, 205$ (32) $\left[\mathrm{M}-\mathrm{NO}_{2}-\mathrm{C}_{3} \mathrm{H}_{6}\right]^{+}, 117$ (100) $\left[\mathrm{C}_{9} \mathrm{H}_{9}\right]^{+}$; HRMS (ESI): calcd for $\mathrm{C}_{19} \mathrm{H}_{20} \mathrm{NO}_{2}{ }^{+}[\mathrm{M}+\mathrm{H}]^{+}$: 294.1488; found: 294.1488.

[1-(tert-Butyl)-3-nitro-2-phenylcyclobutoxy]trimethylsilane $(3 \mathrm{~g})$. The reaction was performed in analogy to the representative procedure for conditions $\mathrm{B}$ (see above) with nitroethene 1a (14.9 mg, $100 \mu \mathrm{mol}, 1.00$ equiv.) and [(3,3-dimethylbut-1-en-2yl)oxy]trimethylsilane (216 $\mu \mathrm{L}, 172 \mathrm{mg}, 1.00 \mathrm{mmol}, 10.0$ equiv.) 
in dichloromethane ( $5 \mathrm{~mL}, c=20 \mathrm{~mm})(t=24 \mathrm{~h})$. Purification by column chromatography $\left(\mathrm{P} / \mathrm{Et}_{2} \mathrm{O}=50 / 1\right)$ yielded $3 \mathrm{~g}$ (12.3 mg, $38.3 \mu \mathrm{mol}, 38 \%$, dr = $77: 23$ ) as a yellow coloured oil. NMR data are given for the major diastereoisomer depicted in Scheme 2. $R_{\mathrm{f}}=0.70\left(\mathrm{P} / \mathrm{Et}_{2} \mathrm{O}=19 / 1\right)$; IR: $\tilde{\nu}\left(\mathrm{cm}^{-1}\right)=3063,3031$, 2958, 1546, 1480, 1395, 1368, 1252, 1146, 1029, 870, 833; ${ }^{1} \mathrm{H}$ NMR (500 MHz, $\left.\mathrm{CDCl}_{3}\right): \delta(\mathrm{ppm})=7.33-7.31\left(\mathrm{~m}, 5 \mathrm{H}, \mathrm{H}_{\mathrm{ar}}\right), 5.13$ (virt. q, $\left.{ }^{3} J \cong{ }^{3} J=8.5 \mathrm{~Hz}, 1 \mathrm{H}, \mathrm{H}-3\right), 4.24\left(\mathrm{~d},{ }^{3} J=8.6 \mathrm{~Hz}, 1 \mathrm{H}, \mathrm{H}-2\right)$, $2.93\left(\mathrm{dd},{ }^{2} J=13.2 \mathrm{~Hz},{ }^{3} J=8.2 \mathrm{~Hz}, 1 \mathrm{H}, \mathrm{CHH}-4\right), 2.50\left(\mathrm{dd},{ }^{2} J=\right.$ $\left.13.2 \mathrm{~Hz},{ }^{3} \mathrm{~J}=8.6 \mathrm{~Hz}, 1 \mathrm{H}, \mathrm{CH} H-4\right), 0.97\left[\mathrm{~s}, 9 \mathrm{H}, \mathrm{C}\left(\mathrm{CH}_{3}\right)_{3}\right], 0.12[\mathrm{~s}$, 9H, OSi $\left.\left.\left(\mathrm{CH}_{3}\right)_{3}\right] ;{ }^{13} \mathrm{C} \mathrm{NMR} \mathrm{(126} \mathrm{MHz,} \mathrm{CDCl}_{3}\right): \delta(\mathrm{ppm})=136.4$ $\left(\mathrm{s}, \mathrm{C}_{\mathrm{ar}}\right), 129.0\left(\mathrm{~d}, 2 \mathrm{C}\right.$, ortho $\left.-\mathrm{C}_{\mathrm{ar}} \mathrm{H}\right), 128.1\left(\mathrm{~d}, 2 \mathrm{C}\right.$, meta $\left.-\mathrm{C}_{\mathrm{ar}} \mathrm{H}\right)$, $127.3\left(\mathrm{~d}\right.$, para $\left.-\mathrm{C}_{\mathrm{ar}} \mathrm{H}\right), 83.3$ (s, C-1), 78.2 (d, C-3), 52.2 (d, C-2), $37.9\left[\mathrm{~s}, \mathrm{C}\left(\mathrm{CH}_{3}\right)_{3}\right], 34.3(\mathrm{t}, \mathrm{C}-4), 25.8\left[\mathrm{q}, 3 \mathrm{C}, \mathrm{C}\left(\mathrm{CH}_{3}\right)_{3}\right], 2.6[\mathrm{q}, 3 \mathrm{C}$, $\left.\mathrm{OSi}\left(\mathrm{CH}_{3}\right)_{3}\right]$; $\mathrm{MS}(\mathrm{EI}): \mathrm{m} / z(\%)=275(17)\left[\mathrm{M}-\mathrm{NO}_{2}\right]^{+}, 219$ (18) $\left[\mathrm{M}-\mathrm{NO}_{2}-\mathrm{C}\left(\mathrm{CH}_{3}\right)_{3}\right]^{+}, 117(100)\left[\mathrm{C}_{9} \mathrm{H}_{9}\right]^{+}, 73(36)\left[\mathrm{Si}\left(\mathrm{CH}_{3}\right)_{3}\right]^{+}$; HRMS (ESI): calcd for $\mathrm{C}_{17} \mathrm{H}_{28} \mathrm{NO}_{3} \mathrm{Si}^{+}[\mathrm{M}+\mathrm{H}]^{+}$: 322.1833; found: 322.1833 .

Trimethyl[(6-nitro-7-phenylbicyclo[3.2.0]heptan-1-yl)oxy] silane $(3 \mathbf{h})$. The reaction was performed in analogy to the representative procedure for conditions $\mathrm{C}$ (see above) with nitroethene 1a $(14.9 \mathrm{mg}, 100 \mu \mathrm{mol}, 1.00$ equiv.) and (cyclopent-1-en-1-yloxy) trimethylsilane (156 mg, $1.00 \mathrm{mmol}, 10.0$ equiv.) in dichloromethane ( $5 \mathrm{~mL}, c=20 \mathrm{~mm})(t=24 \mathrm{~h})$. Purification by column chromatography $\left(\mathrm{P} / \mathrm{Et}_{2} \mathrm{O}=40 / 1\right)$ yielded $3 \mathbf{h}$ (11.7 mg, $38.3 \mu \mathrm{mol}, 38 \%, \mathrm{dr}=61: 39)$ as a colourless oil. NMR data are given for the major diastereoisomer depicted in Scheme 2. $R_{\mathrm{f}}=0.70\left(\mathrm{P} / \mathrm{Et}_{2} \mathrm{O}=9 / 1\right)$; IR: $\tilde{\nu}\left(\mathrm{cm}^{-1}\right)=$ 3004, 2926, 1542, 1497, 1364, 1264, 1045, 1018, 891, 822, 758; ${ }^{1} \mathrm{H}$ NMR $\left(500 \mathrm{MHz}, \mathrm{CDCl}_{3}\right): \delta(\mathrm{ppm})=7.38-7.32(\mathrm{~m}, 2 \mathrm{H}$, meta$\mathrm{H}_{\mathrm{ar}}$ ), 7.29-7.25 (m, 3H, ortho- $\mathrm{H}_{\mathrm{ar}}$, para $\left.-\mathrm{H}_{\mathrm{ar}}\right), 5.35$ (dd, ${ }^{3} J=10.1$ $\mathrm{Hz}, 8.8 \mathrm{~Hz}, 1 \mathrm{H}, \mathrm{H}-6), 4.06$ (d, $\left.{ }^{3} J=8.8 \mathrm{~Hz}, 1 \mathrm{H}, \mathrm{H}-7\right), 3.09$ (virt. t, $\left.{ }^{3} J \cong{ }^{3} J=9.2 \mathrm{~Hz}, 1 \mathrm{H}, \mathrm{H}-5\right), 2.00-1.91\left(\mathrm{~m}, 4 \mathrm{H}, \mathrm{CH}_{2}-3, \mathrm{CH}-2\right.$, $\mathrm{CH}-4), 1.89-1.77$ (m, 1H, CHH-2), 1.62-1.44 (m, 1H, CHH-4), $-0.14\left[\mathrm{~s}, 9 \mathrm{H}, \mathrm{OSi}\left(\mathrm{CH}_{3}\right)_{3}\right] ;{ }^{13} \mathrm{C}$ NMR $\left(126 \mathrm{MHz}, \mathrm{CDCl}_{3}\right): \delta(\mathrm{ppm})$ $=136.5\left(\mathrm{~s}, \mathrm{C}_{\mathrm{ar}}\right), 129.5\left(\mathrm{~d}, 2 \mathrm{C}\right.$, ortho $\left.-\mathrm{C}_{\mathrm{ar}} \mathrm{H}\right), 128.8 / 128.5(\mathrm{~d}, 2 \mathrm{C}$ meta- $\mathrm{C}_{\mathrm{ar}} \mathrm{H}$ ), 127.5/127.3 (d, para- $\left.\mathrm{C}_{\mathrm{ar}} \mathrm{H}\right), 83.5$ (s, C-1), 81.0 (d, C-6), 51.9 (d, C-7), 49.9 (d, C-5), 40.1 (t, $\mathrm{CH}_{2}-2$ ), 26.1 (t, $\mathrm{CH}_{2}-4$ ), $25.9\left(\mathrm{t}, \mathrm{CH}_{2}-3\right), 1.64$ [q, 3C, OSi $\left.\left(\mathrm{CH}_{3}\right)_{3}\right] ; \mathrm{MS}(\mathrm{EI}): \mathrm{m} / z(\%)=259$ (88) $\left[\mathrm{M}-\mathrm{NO}_{2}\right]^{+}, 169(60)\left[\mathrm{M}-\mathrm{NO}_{2}-\mathrm{OSi}\left(\mathrm{CH}_{3}\right)_{3}\right]^{+}, 91$ (28) $\left[\mathrm{C}_{7} \mathrm{H}_{7}\right]^{+}, \quad 73$ (100) $\left[\mathrm{Si}\left(\mathrm{CH}_{3}\right)_{3}\right]^{+} ;$HRMS (ESI): calcd for $\mathrm{C}_{16} \mathrm{H}_{24} \mathrm{NO}_{3} \mathrm{Si}^{+}[\mathrm{M}+\mathrm{H}]^{+}$: 306.1522; found: 306.1522.

1-(Pent-4'-en-1'-yl)-2-(2", 2", 3", 3"-tetramethyl-4"-nitrocyclobutyl)-benzene (2o). A solution of nitroethene $10(21.7 \mathrm{mg}$, $100 \mu \mathrm{mol}, 1.00$ equiv.) and 2,3-dimethyl-2-butene (356 $\mu \mathrm{L}$, $252 \mathrm{mg}, 3.00 \mathrm{mmol}, 30.0$ equiv.) in dichloromethane $(5 \mathrm{~mL}, c=$ $20 \mathrm{~mm}$ ) was irradiated at $\lambda_{\max }=419 \mathrm{~nm}$ for 18 hours at room temperature. Purification by column chromatography $\left(\mathrm{P} / \mathrm{Et}_{2} \mathrm{O}\right.$ $=4 / 1$ ) yielded $20(13.8 \mathrm{mg}, 45.8 \mu \mathrm{mol}, 46 \%)$ as a yellow coloured oil. $R_{\mathrm{f}}=0.68\left(\mathrm{P} / \mathrm{Et}_{2} \mathrm{O}=9 / 1\right)$; IR: $\tilde{\nu}\left(\mathrm{cm}^{-1}\right)=3066,2931$, 2870, 1542, 1460, 1371, 993, 912, 751; ${ }^{1} \mathrm{H}$ NMR $(500 \mathrm{MHz}$, $\left.\mathrm{CDCl}_{3}\right): \delta(\mathrm{ppm})=7.15-7.09(\mathrm{~m}, 3 \mathrm{H}, \mathrm{H}-3, \mathrm{H}-4, \mathrm{H}-5), 7.07-7.03$ (m, 1H, H-6), 5.83 (ddt, ${ }^{3} J=17.0 \mathrm{~Hz}, 10.2 \mathrm{~Hz}, 6.7 \mathrm{~Hz}, 1 \mathrm{H}, \mathrm{H}-4^{\prime}$ ), 5.02 (virt. dq, ${ }^{3} J=17.2 \mathrm{~Hz},{ }^{2} J \cong{ }^{4} J=1.7 \mathrm{~Hz}, 1 \mathrm{H}, \mathrm{CH}-5^{\prime}$ ), 4.98-4.92 (m, 2H, CHH-5', H-4"), 4.15 (d, ${ }^{3} \mathrm{~J}=10.2 \mathrm{~Hz}, 1 \mathrm{H}$, H-1"), 2.76 (ddd, ${ }^{2} J=14.0 \mathrm{~Hz},{ }^{3} J=10.5 \mathrm{~Hz}, 5.3 \mathrm{~Hz}, 1 \mathrm{H}$,
$\mathrm{CHH}-1^{\prime}$ ), 2.46 (ddd, ${ }^{2} J=14.0 \mathrm{~Hz},{ }^{3} J=10.6 \mathrm{~Hz}, 6.0 \mathrm{~Hz}, 1 \mathrm{H}, \mathrm{CH} \mathrm{H}^{-}$ $\left.1^{\prime}\right)$, 2.19-2.03 (m, 2H, H-3'), 1.82-1.72 (m, 1H, CHH-2'), 1.70-1.58 (m, 1H, CHH-2'), 1.18 (s, 3H, $\left.\mathrm{CH}_{3}-3^{\prime \prime}\right), 1.10(\mathrm{~s}, 3 \mathrm{H}$, $\left.\mathrm{CH}_{3}-3^{\prime \prime}\right), 1.09$ (s, 3H, $\left.\mathrm{CH}_{3}-2^{\prime \prime}\right), 0.67$ (s, 3H, $\left.\mathrm{CH}_{3}-2^{\prime \prime}\right) ;{ }^{13} \mathrm{C} \mathrm{NMR}$ $\left(126 \mathrm{MHz}, \mathrm{CDCl}_{3}\right): \delta(\mathrm{ppm})=141.7(\mathrm{~s}, \mathrm{C}-1), 138.6\left(\mathrm{~d}, \mathrm{C}-4^{\prime}\right)$, 133.2 (s, C-2), 129.8 (d, C-6), 127.1 (d, C-5)*, 127.0 (d, C-3), $125.9(\mathrm{~d}, \mathrm{C}-4)^{*}, 115.3$ (t, C-5'), 85.3 (d, C-4"), 45.6 (d, C-1"), 44.4 (s, C-2"), 40.1 (s, C-3"), 33.8 (t, C-3'), 32.4 (t, C-1'), 30.6 (t, C-2'), 24.6 [q, $\left.\left(\mathrm{C}-2^{\prime \prime}\right) \mathrm{CH}_{3}\right], 22.8$ [q, $\left.\left(\mathrm{C}-3^{\prime \prime}\right) \mathrm{CH}_{3}\right], 21.8$ [q, $\left(\mathrm{C}-2^{\prime \prime}\right) \mathrm{CH}_{3}$ ], $19.4\left[\mathrm{q},\left(\mathrm{C}-3^{\prime \prime}\right) \mathrm{CH}_{3}\right]$ (* the assignments are interconvertible); MS (EI): $m / z(\%)=301(2)[\mathrm{M}]^{+}, 255(20)\left[\mathrm{M}-\mathrm{NO}_{2}\right]^{+}, 199$ (49) $\left[\mathrm{M}-\mathrm{NO}_{2}-\mathrm{C}_{3} \mathrm{H}_{6}\right]^{+}, 143(100)\left[\mathrm{C}_{11} \mathrm{H}_{11}\right]^{+}$; HRMS (ESI): calcd for $\mathrm{C}_{19} \mathrm{H}_{27} \mathrm{NO}_{2}{ }^{+}[\mathrm{M}+\mathrm{H}]^{+}$: 302.2115; found: 302.2115.

\section{2,2,3,3-Tetramethyl-4-phenylcyclobutan-1-amine} According to a literature known procedure: ${ }^{22} \mathrm{Zn}$ powder (350 mg, $5.36 \mathrm{mmol}, 25.0$ equiv.) was added in small portions to a stirred solution of nitrocyclobutane $2 \mathrm{a}(50.0 \mathrm{mg}$, $214 \mu \mathrm{mol}, 1.00$ equiv.) in a mixture of water/acetic acid $(2 \mathrm{~mL}$; $1 / 1 \mathrm{v} / \mathrm{v})$. The suspension was stirred for four hours at room temperature. Aqueous $\mathrm{NaOH}$ solution $(c=5 \mathrm{M})$ was added until $\mathrm{pH}=7$ was reached. The solution was extracted with dichloromethane $(2 \times 50 \mathrm{~mL})$. The combined organic layers were washed with saturated aqueous $\mathrm{NaCl}$ solution $(100 \mathrm{~mL})$, dried over $\mathrm{Na}_{2} \mathrm{SO}_{4}$, filtered and concentrated in vacuo to yield 4 (33.6 mg, $165 \mu \mathrm{mol}, 77 \%)$ as a colourless oil. IR: $\tilde{\nu}\left(\mathrm{cm}^{-1}\right)=$ 3060, 2959, 2866, 2604, 1566, 1458, 1449, 1358, 1337, 1270, 1132, 885, 810; ${ }^{1} \mathrm{H}$ NMR (400 $\left.\mathrm{MHz}, \mathrm{CDCl}_{3}\right): \delta(\mathrm{ppm})=$ 7.29-7.21 (m, 2H, meta $\left.-\mathrm{H}_{\mathrm{Ph}}\right), 7.19-7.08\left(\mathrm{~m}, 3 \mathrm{H}\right.$, ortho $-\mathrm{H}_{\mathrm{Ph}}$, para $\left.-\mathrm{H}_{\mathrm{Ph}}\right), 3.37$ (d, $\left.{ }^{3} \mathrm{~J}=9.9 \mathrm{~Hz}, 1 \mathrm{H}, \mathrm{H}-1\right), 2.86\left(\mathrm{~d},{ }^{3} \mathrm{~J}=9.9 \mathrm{~Hz}\right.$, $1 \mathrm{H}, \mathrm{H}-4), 1.51$ (br. s, 2H, $\mathrm{NH}_{2}$ ), 1.03 (s, 3H, $\left.\mathrm{CH}_{3}-2\right), 1.01$ (s, 3H, $\left.\mathrm{CH}_{3}-3\right), 0.93\left(\mathrm{~s}, 3 \mathrm{H}, \mathrm{CH}_{3}-2\right), 0.59$ (s, 3H, $\left.\mathrm{CH}_{3}-3\right) ;{ }^{13} \mathrm{C} \mathrm{NMR}$ $\left(101 \mathrm{MHz}, \mathrm{CDCl}_{3}\right): \delta(\mathrm{ppm})=139.6\left(\mathrm{~s}, \mathrm{C}_{\mathrm{Ph}}\right), 128.3(\mathrm{~d}, 2 \mathrm{C}$, meta$\left.\mathrm{C}_{\mathrm{Ph}} \mathrm{H}\right), 127.7$ (d, 2C, ortho $\left.-\mathrm{C}_{\mathrm{Ph}} \mathrm{H}\right), 126.1\left(\mathrm{~d}\right.$, para $\left.-\mathrm{C}_{\mathrm{Ph}} \mathrm{H}\right), 56.8(\mathrm{~d}$, C-4), 55.5 (d, C-1), 41.7 (s, C-2), 39.6 (s, C-3), 24.2 [q, (C-3) $\mathrm{CH}_{3}$ ], 22.6 [q, (C-2) $\left.\mathrm{CH}_{3}\right], 21.1$ [q, (C-3) $\left.\mathrm{CH}_{3}\right], 18.7$ [q, (C-2)CH $\mathrm{CH}_{3}$; MS (EI, $70 \mathrm{eV}): m / z(\%)=132(5)\left[\mathrm{M}-\mathrm{C}_{4} \mathrm{H}_{9} \mathrm{~N}\right]^{+}, 119(100)[\mathrm{M}-$ $\left.\mathrm{C}_{4} \mathrm{H}_{9} \mathrm{~N}-\mathrm{CH}_{3}\right]^{+}, 91(13)\left[\mathrm{C}_{7} \mathrm{H}_{7}\right]^{+}, 71(31)\left[\mathrm{C}_{4} \mathrm{H}_{9}\right]^{+}, 56$ (11); HRMS (ESI): calcd for $\mathrm{C}_{14} \mathrm{H}_{22} \mathrm{~N}^{+}[\mathrm{M}+\mathrm{H}]^{+}$: 204.1741; found: 204.1748 .

(2',2'-Dicyclopropyl-4'-nitrocyclobutyl)-benzene (3i) and 1-nitro-3-propylidene-2,3,3a,4,5,9b-hexahydro- $1 \mathrm{H}$-cyclopenta[a] naphthalene (7). A solution of nitroethene 1a (14.9 mg, $100 \mu \mathrm{mol}, 1.00$ equiv.) and 1,1-dicyclopropyl-ethylene (109 mg, $1.00 \mathrm{mmol}, 10.0$ equiv.) in dichloromethane ( $5 \mathrm{~mL}, c=20 \mathrm{~mm}$ ) was irradiated at $\lambda_{\max }=419 \mathrm{~nm}$ for twelve hours at room temperature. Purification by column chromatography $\left(\mathrm{P} / \mathrm{Et}_{2} \mathrm{O}=40\right.$ / 1) yielded $3 \mathbf{i}(18.0 \mathrm{mg}, 69.9 \mu \mathrm{mol}, 70 \%)$ and $7(2.20 \mathrm{mg}$, $8.55 \mu \mathrm{mol}, 9 \%)$ both as a yellow coloured oil. $3 \mathrm{i}: R_{\mathrm{f}}=0.58(\mathrm{P} /$ $\left.\mathrm{Et}_{2} \mathrm{O}=19 / 1\right)$; IR: $\tilde{\nu}\left(\mathrm{cm}^{-1}\right)=3079,3004,1542,1449,1369,1017$, 822, 758; ${ }^{1} \mathrm{H}$ NMR (400 MHz, $\left.\mathrm{CDCl}_{3}\right): \delta(\mathrm{ppm})=7.44-7.20(\mathrm{~m}$, $5 \mathrm{H}, \mathrm{H}_{\mathrm{ar}}$ ), 5.20 (virt. q, $\left.{ }^{3} \mathrm{~J} \cong{ }^{3} J=8.7 \mathrm{~Hz}, 1 \mathrm{H}, \mathrm{H}-4^{\prime}\right), 3.97\left(\mathrm{~d},{ }^{3} J=\right.$ $\left.9.2 \mathrm{~Hz}, 1 \mathrm{H}, \mathrm{H}-1^{\prime}\right), 2.06\left(\mathrm{dd},{ }^{2} J=12.2 \mathrm{~Hz},{ }^{3} \mathrm{~J}=8.7 \mathrm{~Hz}, 1 \mathrm{H}\right.$, $\mathrm{CH}-\mathrm{H}^{\prime}$ ), 1.71 (dd, ${ }^{3} J=12.2 \mathrm{~Hz},{ }^{3} J=8.3 \mathrm{~Hz}, 1 \mathrm{H}, \mathrm{CH} H-3^{\prime}$ ), 1.09 $\left[\mathrm{tt},{ }^{3} \mathrm{~J}=8.4 \mathrm{~Hz}, 5.5 \mathrm{~Hz}, 1 \mathrm{H}, \mathrm{CH}\left(\mathrm{CH}_{2}\right)_{2}\right], 0.60-0.25[\mathrm{~m}, 6 \mathrm{H}$, $\left.\mathrm{CH}\left(\mathrm{CH}_{2}\right)_{2}, \mathrm{CH}\left(\mathrm{CH}_{2}\right)_{2}\right], 0.20-0.13\left[\mathrm{~m}, 1 \mathrm{H}, \mathrm{CH}\left(\mathrm{CH}_{2}\right)_{2}\right] ;{ }^{13} \mathrm{C} \mathrm{NMR}$ $\left(101 \mathrm{MHz}, \mathrm{CDCl}_{3}\right): \delta(\mathrm{ppm})=136.4\left(\mathrm{~s}, \mathrm{C}_{\mathrm{ar}}\right), 128.5(\mathrm{~d}, 2 \mathrm{C}$, meta$\left.\mathrm{C}_{\mathrm{ar}} \mathrm{H}\right), 127.7$ (d, 2C, ortho- $\left.\mathrm{C}_{\mathrm{ar}} \mathrm{H}\right), 127.2$ (d, para $\left.-\mathrm{C}_{\mathrm{ar}} \mathrm{H}\right), 76.6$ (d, 
C-4'), 55.5 (d, C-1'), 41.7 (s, C-2'), $27.8\left(\mathrm{t}, \mathrm{C}-3^{\prime}\right), 20.2[\mathrm{~d}, \mathrm{CH}$ $\left.\left(\mathrm{CH}_{2}\right)_{2}\right], 14.6\left[\mathrm{~d}, \mathrm{CH}\left(\mathrm{CH}_{2}\right)_{2}\right], 1.93\left[\mathrm{t}, \mathrm{CH}\left(\mathrm{CH}_{2}\right)_{2}\right], 1.74[\mathrm{t}, \mathrm{CH}$ $\left.\left(\mathrm{CH}_{2}\right)_{2}\right], 0.90\left[\mathrm{t}, \mathrm{CH}\left(\mathrm{CH}_{2}\right)_{2}\right], 0.72\left[\mathrm{t}, \mathrm{CH}\left(\mathrm{CH}_{2}\right)_{2}\right]$; $\mathrm{MS}(\mathrm{EI}): \mathrm{m} / \mathrm{z}(\%)$ $=211$ (4) $\left[\mathrm{M}-\mathrm{NO}_{2}\right]^{+}, 169$ (16) $\left[\mathrm{M}-\mathrm{NO}_{2}-\mathrm{C}_{3} \mathrm{H}_{6}\right]^{+}, 117$ (100) $\left[\mathrm{C}_{9} \mathrm{H}_{9}\right]^{+}$; HRMS (ESI): calcd for $\mathrm{C}_{16} \mathrm{H}_{20} \mathrm{NO}_{2}{ }^{+}[\mathrm{M}+\mathrm{H}]^{+}$: 258.1448; found: 258.1449. 7: $R_{\mathrm{f}}=0.69\left(\mathrm{P} / \mathrm{Et}_{2} \mathrm{O}=19 / 1\right)$; IR: $\tilde{\nu}\left(\mathrm{cm}^{-1}\right)=$ 3419, 2928, 1722, 1547, 1367, 1023, 856, 791; ${ }^{1} \mathrm{H}$ NMR $\left(500 \mathrm{MHz}, \mathrm{CDCl}_{3}\right): \delta(\mathrm{ppm})=7.17-7.05\left(\mathrm{~m}, 4 \mathrm{H}, \mathrm{H}_{\mathrm{ar}}\right), 5.45(\mathrm{ttd}$, ${ }^{3} J=7.1 \mathrm{~Hz},{ }^{4} J=2.5 \mathrm{~Hz}, 1.6 \mathrm{~Hz}, 1 \mathrm{H}, \mathrm{C}=\mathrm{CHCH}_{2} \mathrm{CH}_{3}$ ), 4.90 (virt. $\mathrm{q},{ }^{3} J \cong{ }^{3} J=7.3 \mathrm{~Hz}, 1 \mathrm{H}, \mathrm{H}-1$ ), 3.90 (virt. $\mathrm{t},{ }^{3} J \cong{ }^{3} J=7.5 \mathrm{~Hz}, 1 \mathrm{H}$, H-9b), 3.07-2.94 (m, 2H, H-3a, CHH-2), 2.90 (dddd, ${ }^{2} J=17.3$ $\left.\mathrm{Hz},{ }^{3} J=7.9 \mathrm{~Hz},{ }^{4} J=2.7 \mathrm{~Hz}, 1.4 \mathrm{~Hz}, 1 \mathrm{H}, \mathrm{CH} H-2\right), 2.80-2.75$ (m, $1 \mathrm{H}, \mathrm{CH}-5)$, 2.73-2.68 (m, 1H, CHH-5), 2.07-1.98 (m, 2H, $\left.\mathrm{C}=\mathrm{CHCH}_{2} \mathrm{CH}_{3}\right), 1.84\left(\mathrm{ddt},{ }^{2} \mathrm{~J}=13.8 \mathrm{~Hz},{ }^{3} \mathrm{~J}=6.2 \mathrm{~Hz}, 4.7 \mathrm{~Hz}, 1 \mathrm{H}\right.$, $\mathrm{CHH}-4), 1.67$ (dtd, ${ }^{2} J=13.8 \mathrm{~Hz},{ }^{3} J=9.5 \mathrm{~Hz}, 4.8 \mathrm{~Hz}, 1 \mathrm{H}, \mathrm{CH} H-$ 4), $1.00\left(\mathrm{t},{ }^{3} \mathrm{~J}=7.5 \mathrm{~Hz}, 3 \mathrm{H}, \mathrm{CH}_{3}\right) ;{ }^{13} \mathrm{C} \mathrm{NMR}\left(126 \mathrm{MHz}, \mathrm{CDCl}_{3}\right): \delta$ $(\mathrm{ppm})=138.7(\mathrm{~s}, \mathrm{C}-3), 137.1(\mathrm{~s}, \mathrm{C}-5 \mathrm{a}), 134.4(\mathrm{~s}, \mathrm{C}-9 \mathrm{a}), 129.3(\mathrm{~d}$, C-6), 128.6 (d, C-9), 127.2 (d, C-8), 126.6 (d, C-7), 125.9 (d, $C=\mathrm{CHCH}_{2} \mathrm{CH}_{3}$ ), 92.2 (d, C-1), 47.7 (d, C-9b), 42.0 (d, C-3a), 34.6 (t, C-2), 27.8 (t. C-5), 27.3 (t, C-4), 22.8 (t, C= $=\mathrm{CHCH}_{2} \mathrm{CH}_{3}$ ), 14.1 (q, $\left.\mathrm{CH}_{3}\right)$; $\mathrm{MS}(\mathrm{EI}): m / z(\%)=181(100)\left[\mathrm{M}-\mathrm{NO}_{2}-\mathrm{C}_{2} \mathrm{H}_{4}\right]^{+}$, 167 (40) $\left[\mathrm{C}_{13} \mathrm{H}_{11}\right]^{+}, 128$ (16) $\left[\mathrm{C}_{10} \mathrm{H}_{8}\right]^{+}$; HRMS (ESI): calcd for $\mathrm{C}_{16} \mathrm{H}_{20} \mathrm{NO}_{2}^{+}[\mathrm{M}+\mathrm{H}]^{+}$: 258.1448; found: 258.1449.

Methyl (E)-1-nitro-3-propylidene-2,3,3a,4,5,9b-hexahydro-1 $H$ cyclopenta[a]naphthalene-7-carboxylate (8). Colourless solid. $R_{\mathrm{f}}=0.53\left(\mathrm{P} / \mathrm{Et}_{2} \mathrm{O}=19 / 1\right)$; IR: $\tilde{\nu}\left(\mathrm{cm}^{-1}\right)=3423,2955,1720,1550$, 1437, 1368, 1284, 1105, 762; ${ }^{1} \mathrm{H}$ NMR (500 MHz, $\mathrm{CDCl}_{3}$ ): $\delta$ $(\mathrm{ppm})=7.80-7.77(\mathrm{~m}, 2 \mathrm{H}, \mathrm{H}-6, \mathrm{H}-8), 7.14\left(\mathrm{~d},{ }^{3} \mathrm{~J}=8.6 \mathrm{~Hz}, 1 \mathrm{H}\right.$, $\mathrm{H}-9$ ), 5.46 (virt. tq, ${ }^{3} J=6.9 \mathrm{~Hz},{ }^{4} J \cong{ }^{4} J=2.4 \mathrm{~Hz}, 1 \mathrm{H}$, $\mathrm{C}=\mathrm{CHCH}_{2} \mathrm{CH}_{3}$ ), 4.88 (virt. q, ${ }^{3} J \cong{ }^{3} J=7.3 \mathrm{~Hz}, 1 \mathrm{H}, \mathrm{H}-1$ ), 3.96-3.91 (m, 1H, H-9b), 3.90 (s, 3H, $\left.\mathrm{CO}_{2} \mathrm{CH}_{3}\right), 3.11-2.95(\mathrm{~m}$, $2 \mathrm{H}, \mathrm{H}-3 \mathrm{a}, \mathrm{CH}-2), 2.95-2.80$ (m, 2H, $\mathrm{CH}-2, \mathrm{CH}-5)$, 2.78-2.66 (m, 1H, CHH-5), 2.08-1.96 (m, 2H, C= $\mathrm{CHCH}_{2} \mathrm{CH}_{3}$ ), 1.91-1.81 (m, 1H, CHH-4), 1.68 (dtd, ${ }^{2} J=13.9 \mathrm{~Hz},{ }^{3} J=9.3 \mathrm{~Hz}$, $4.8 \mathrm{~Hz}, 1 \mathrm{H}, \mathrm{CH} H-4$ ), 1.00 (t, ${ }^{3} J=7.5 \mathrm{~Hz}, 3 \mathrm{H}, \mathrm{C}=\mathrm{CHCH}_{2} \mathrm{CH}_{3}$ ); ${ }^{13} \mathrm{C}$ NMR (126 MHz, $\left.\mathrm{CDCl}_{3}\right): \delta(\mathrm{ppm})=138.7(\mathrm{~s}, \mathrm{C}-3), 137.1(\mathrm{~s}$, C-5a), 134.4 (s, C-9a), 129.3 (d, C-6), 128.6 (d, C-9), 127.2 (d, C-8), 126.6 (d, C-7), 125.9 (d, $C=\mathrm{CHCH}_{2} \mathrm{CH}_{3}$ ), 92.2 (d, C-1), $52.3\left(\mathrm{q}, \mathrm{CO}_{2} \mathrm{CH}_{3}\right) 47.7$ (d, C-9b), 42.0 (d, C-3a), 34.6 (t, C-2), 27.8 (t. C-5), 27.3 (t, C-4), 22.8 (t, $\mathrm{C}=\mathrm{CHCH}_{2} \mathrm{CH}_{3}$ ), 14.1 (q, $\left.\mathrm{C}=\mathrm{CHCH}_{2} \mathrm{CH}_{3}\right) ; \mathrm{MS}(\mathrm{EI}): \mathrm{m} / z(\%)=284$ (19) $\left[\mathrm{M}-\mathrm{OCH}_{3}\right]^{+}, 253$ (54), 149 (100) $\left[\mathrm{C}_{9} \mathrm{H}_{9} \mathrm{O}_{2}\right]^{+}, 115$ (49), 91 (63) [ $\left.\mathrm{C}_{7} \mathrm{H}_{7}\right]^{+}$; HRMS (ESI): calcd for $\mathrm{C}_{18} \mathrm{H}_{22} \mathrm{NO}_{2}{ }^{+}[\mathrm{M}+\mathrm{H}]^{+}$: 316.1543; found: 316.1545 .

(E)-1-Nitro-3-( propylidene-3- $d$ )-2,3,3a,4,5,9b-hexahydro-1Hcyclopenta $[a]$ naphthalene-6,7,8,9- $\boldsymbol{d}_{\mathbf{4}}\left(\mathbf{7}-\boldsymbol{d}_{\mathbf{5}}\right)$. Yellow coloured oil. $R_{\mathrm{f}}=0.70\left(\mathrm{P} / \mathrm{Et}_{2} \mathrm{O}=19 / 1\right)$; IR: $\tilde{\nu}\left(\mathrm{cm}^{-1}\right)=3418,2928,1711,1547$, 1368, 1261, 1024, 858, 803, 752; ${ }^{1} \mathrm{H}$ NMR (400 $\mathrm{MHz}, \mathrm{CDCl}_{3}$ ): $\delta(\mathrm{ppm})=5.45\left(\mathrm{tq},{ }^{3} J=7.1 \mathrm{~Hz},{ }^{4} J=2.4 \mathrm{~Hz}, 1 \mathrm{H}, \mathrm{C}=\mathrm{CHCH}_{2} \mathrm{CH}_{3}\right)$, 4.90 (virt. q, ${ }^{3} \mathrm{~J} \cong{ }^{3} J=7.4 \mathrm{~Hz}, 1 \mathrm{H}, \mathrm{H}-1$ ), 3.90 (virt. $\mathrm{t},{ }^{3} \mathrm{~J} \cong{ }^{3} \mathrm{~J}=7.6$ $\mathrm{Hz}, 1 \mathrm{H}, \mathrm{H}-9 \mathrm{~b}), 3.08-2.94$ (m, 2H, H-3a, CHH-2), 2.90 (dd, ${ }^{2} J=$ $\left.17.3 \mathrm{~Hz},{ }^{3} J=7.8 \mathrm{~Hz}, 1 \mathrm{H}, \mathrm{CH} H-2\right), 2.81-2.75$ (m, 1H, CHH-5), 2.69 (ddd, ${ }^{2} J=16.4 \mathrm{~Hz},{ }^{3} J=9.1 \mathrm{~Hz}, 4.8 \mathrm{~Hz}, 1 \mathrm{H}, \mathrm{CH} H-5$ ), 2.05-1.98 (m, 2H, C= $\left.\mathrm{CHCH}_{2} \mathrm{CH}_{3}\right), 1.89-1.79(\mathrm{~m}, 1 \mathrm{H}, \mathrm{CHH}-4)$, $1.67\left(\mathrm{dtd},{ }^{2} \mathrm{~J}=14.0 \mathrm{~Hz},{ }^{3} \mathrm{~J}=9.4 \mathrm{~Hz}, 4.8 \mathrm{~Hz}, 1 \mathrm{H}, \mathrm{CH}-4\right), 0.98(\mathrm{tt}$, $\left.{ }^{3} J=7.6 \mathrm{~Hz},{ }^{2} J=2.1 \mathrm{~Hz}, 2 \mathrm{H}, \mathrm{CH}_{2} \mathrm{D}\right) ;{ }^{13} \mathrm{C} \mathrm{NMR}(101 \mathrm{MHz}$,
$\left.\mathrm{CDCl}_{3}\right): \delta(\mathrm{ppm})=138.8(\mathrm{~s}, \mathrm{C}-3), 137.0(\mathrm{~s}, \mathrm{C}-5 \mathrm{a}), 134.4(\mathrm{~s}, \mathrm{C}-9 \mathrm{a})$, 126.0 (d, $C=\mathrm{CHCH}_{2} \mathrm{CH}_{2} \mathrm{D}$ ), 92.2 (d, C-1), 47.6 (d, C-9b), 42.1 (d, C-3a), 34.6 (t, C-2), 27.7 (t. C-5), 27.3 (t, C-4), 22.8 (t, $\left.\mathrm{C}=\mathrm{CHCH}_{2} \mathrm{CH}_{2} \mathrm{D}\right), 14.1\left(\mathrm{t},{ }^{1} J_{\mathrm{CD}}=19.4 \mathrm{~Hz}, \mathrm{C}=\mathrm{CHCH}_{2} \mathrm{CH}_{2} \mathrm{D}\right)$ (the aromatic signals of carbon atoms linked to deuterium atoms were not visible in the ${ }^{13} \mathrm{C}$-NMR spectrum); $\mathrm{MS}$ (EI): $\mathrm{m} / \mathrm{z}$ $(\%)=215(65)\left[\mathrm{M}-\mathrm{NO}_{2}\right]^{+}, 185(100)\left[\mathrm{M}-\mathrm{NO}_{2}-\mathrm{C}_{2} \mathrm{H}_{4}\right]^{+}, 171$ (36), 132 (20) $\left[\mathrm{C}_{10} \mathrm{H}_{4} \mathrm{D}_{4}\right]^{+}, 95$ (6) $\left[\mathrm{C}_{7} \mathrm{H}_{3} \mathrm{D}_{4}\right]^{+}$; HRMS (ESI): calcd for $\mathrm{C}_{16} \mathrm{H}_{15} \mathrm{D}_{5} \mathrm{NO}_{2}{ }^{+}[\mathrm{M}+\mathrm{H}]^{+}$: 263.1802; found: 263.1804 .

(3'-Methyl-4'-nitrocyclobutane)-1,2-diyl-dibenzene $\quad\left(3 \mathbf{j} / 3 \mathbf{j}^{\prime}\right)$. General procedure for the $[2+2]$ photocycloaddition of $\mathbf{1 a}$ to trans- $\beta$-methylstyrene: a solution of nitroethene 1 a $(29.8 \mathrm{mg}$, $200 \mu \mathrm{mol}, 1.00$ equiv.) and trans- $\beta$-methylstyrene $(259 \mu \mathrm{L}$, $236 \mathrm{mg}, 2.00 \mathrm{mmol}, 10.0$ equiv. $)$ in dichloromethane $(5 \mathrm{~mL}, c=$ $20 \mathrm{~mm}$ ) was irradiated at $\lambda_{\max }=419 \mathrm{~nm}$ for twelve hours at room temperature. Purification by column chromatography $(\mathrm{P} /$ $\left.\mathrm{Et}_{2} \mathrm{O}=50 / 1\right)$ yielded $3 \mathbf{j} / 3 \mathbf{j}^{\prime}(28.1 \mathrm{mg}, 1.05 \mathrm{mmol}, 53 \%$, dr $=$ $46: 54)$ as a colourless oil. Starting material was recovered as cis-isomer cis-1a (6.60 mg, $42.3 \mu \mathrm{mol}, 22 \%)$. General procedure for the $[2+2]$ photocycloaddition of $\mathbf{1 a}$ to cis- $\beta$-methylstyrene: a solution of nitroethene ( $29.8 \mathrm{mg}, 200 \mu \mathrm{mol}, 1.00$ equiv.) and cis- $\beta$-methylstyrene ( $260 \mu \mathrm{L}, 236 \mathrm{mg}, 2.00 \mathrm{mmol}, 10.0$ equiv.) in dichloromethane $(5 \mathrm{~mL}, c=20 \mathrm{~mm})$ was irradiated at $\lambda_{\max }=$ $419 \mathrm{~nm}$ for twelve hours at room temperature. Purification by column chromatography $\left(\mathrm{P} / \mathrm{Et}_{2} \mathrm{O}=50 / 1\right)$ yielded $3 \mathbf{j} / 3 \mathbf{j}^{\prime}$ (45.8 mg, $1.12 \mathrm{mmol}, 56 \%$, dr $=77: 23$ ) as a colourless oil. Starting material was recovered as cis-isomer cis-1a $(6.80 \mathrm{mg}$, $45.6 \mu \mathrm{mol}, 23 \%)$. The analytical data obtained matched those reported in the literature. ${ }^{13}$

\section{Conflicts of interest}

There are no conflicts to declare.

\section{Acknowledgements}

Financial support by the European Research Council under the European Union's Horizon 2020 research and innovation programme (grant agreement No. 665951 - ELICOS) is gratefully acknowledged.

\section{Notes and references}

1 B. Priebs, Justus Liebigs Ann. Chem., 1884, 225, 319-364.

2 J. Meisenheimer and F. Heim, Justus Liebigs Ann. Chem., 1907, 355, 260-267.

3 D. B. Miller, P. W. Flanagan and H. Shechter, J. Am. Chem. Soc., 1972, 94, 3912-3918.

4 G. R. Desiraju and V. R. Pedireddi, J. Chem. Soc., Chem. Commun., 1989, 1112-1113.

5 O. L. Chapman, A. A. Griswold, E. Hoganson, G. Lenz and J. Reasoner, Pure Appl. Chem., 1964, 9, 585-590.

6 E. D. Hoganson, Ph.D. thesis, Iowa State University, 1965. 
7 T. Majima, C. Pac and H. Sakurai, J. Am. Chem. Soc., 1980, 102, 5265-5273.

8 D. Ramkumar and S. Sankararaman, J. Chem. Soc., Perkin Trans. 2, 1996, 939-941.

9 A. A. Russell, O. L. Chapman, J. T. Magner and M. Selke, J. Chem. Educ., 1996, 73, 854-856.

10 S. M. Stevenson, R. F. Higgins, M. P. Shores and E. M. Ferreira, Chem. Sci., 2017, 8, 654-660.

11 Review: S. Poplata, A. Tröster, Y.-Q. Zou and T. Bach, Chem. Rev., 2016, 116, 9748-9815.

12 Recent examples: (a) S. Stegbauer, C. Jandl and T. Bach, Angew. Chem., Int. Ed., 2018, 57, 14593-14596; (b) A. HölzlHobmeier, A. Bauer, A. V. Silva, S. M. Huber, C. Bannwarth and T. Bach, Nature, 2018, 564, 240-243.

13 L. Mohr and T. Bach, Synlett, 2017, 28, 2946-2950.

14 L. Henry, Comptes Rendus, 1895, 120, 1265-1268.

15 M. Sathish, J. Chetna, N. H. Krishna, N. Shankaraiah, A. Alarifi and A. Kamal, J. Org. Chem., 2016, 81, 2159-2165.

16 (a) E. A. Braude, E. R. H. Jones and G. G. Rose, J. Chem. Soc., 1947, 1104-1105; (b) D. J. Cowley, J. Chem. Soc., Perkin Trans. 2, 1975, 1576-1580.

17 (a) A. L. Bluhm and J. Weinstein, J. Am. Chem. Soc., 1965, 87, 5511-5512; (b) J. A. Sousa, J. Weinstein and A. L. Bluhm, J. Org. Chem., 1969, 34, 3320-3323; (c) D. B. Miller, P. W. Flanagan and H. Shechter, J. Org. Chem., 1976, 41, 2112-2120; (d) M. Z. Kassaee and E. Vessally, J. Photochem. Photobiol., A, 2005, 172, 331-336.

18 A. Nandi, R. Ghosh and D. K. Palit, J. Photochem. Photobiol., $A, 2016,321,171-179$ and references cited therein.

19 S. Chandrasekhar, B. Tiwari, B. B. Parida and C. R. Reddy, Tetrahedron: Asymmetry, 2008, 19, 495-499.

20 Nitroethene 10 was prepared from the corresponding aldehyde (M. M. Coulter, P. K. Dornan and V. M. Dong, J. Am. Chem. Soc., 2009, 131, 6932-6933) in a Henry reaction.

21 (a) G. W. Kabalka and R. S. Varma, Org. Prep. Proced. Int., 1987, 19, 283-328; (b) R. Ballini, E. Marcantoni and M. Petrini, in Amino Group Chemistry: From Synthesis to the Life Sciences, ed. A. Ricci, Wiley-VCH, Weinheim, 2008, pp. 93-148.

22 G. Talavera, E. Reyes, J. L. Vicario and L. Carrillo, Angew. Chem., Int. Ed., 2012, 51, 4104-4107.

23 (a) A. Barański and E. Cholewka, Chem. Pap., 1991, 45, 449455; (b) S.-Q. Zhang, H.-G. Wang, K.-M. Pei, X. Zheng and D. L. Phillips, J. Chem. Phys., 2007, 126, 194505.

24 S. Ahuja, R. Raghunathan, E. Kumarasamy, S. Jockusch and J. Sivaguru, J. Am. Chem. Soc., 2018, 140, 13185-13189 and references cited therein.
25 (a) A. Rudolph and A. C. Weedon, Can. J. Chem., 1990, 68, 1590-1597; (b) S. Hu and D. C. Neckers, J. Org. Chem., 1997, 62, 755-757; (c) C. Y. Gan and J. N. Lambert, J. Chem. Soc., Perkin Trans. 1, 1998, 2363-2372; (d) S. C. Coote, A. Pöthig and T. Bach, Chem. - Eur. J., 2015, 21, 69066912.

26 G. Hu, J. Xu and P. Li, Org. Lett., 2014, 16, 6036-6039.

27 (a) W. L. Dilling, T. E. Tabor, F. P. Boer and P. P. North, J. Am. Chem. Soc., 1970, 92, 1399-1400; (b) N. P. Peet, R. L. Cargill and D. F. Bushey, J. Org. Chem., 1973, 38, 1218-1221; (c) N. C. Yang, M. Kimura and W. Eisenhardt, J. Am. Chem. Soc., 1973, 95, 5058-5060; (d) J. F. D. Kelly, J. M. Kelly and T. B. H. McMurry, J. Chem. Soc., Perkin Trans. 2, 1999, 1933-1941.

28 (a) D. Rehm and A. Weller, Isr. J. Chem., 1970, 8, 259-271; (b) D. M. Arias-Rotondo and J. K. McCusker, Chem. Soc. Rev., 2016, 45, 5803-5820; (c) L. Buzzetti, G. E. M. Crisenza and P. Melchiorre, Angew. Chem., Int. Ed., 2019, 58, 37303747.

29 All redox potentials are given as potentials against a saturated calomel electrode (SCE). For the redox potential of 1a, see: J. A. Squella, J. C. Sturm, B. Weiss-Lopez, M. Bontá and L. J. Núñez-Vergara, J. Electroanal. Chem., 1999, 466, 90-98.

30 M. Patz, H. Mayr, J. Maruta and S. Fukuzumi, Angew. Chem., Int. Ed., 1995, 34, 1225-1227.

31 C. Huo, X. Jia, W. Zhang, L. Yang, J. Lü and Z.-L. Liu, Synlett, 2004, 251-254.

32 S. Fukuzumi, M. Fujita, J. Otera and Y. Fujita, J. Am. Chem. Soc., 1992, 114, 10271-10278.

33 N. P. Schepp and L. J. Johnston, J. Am. Chem. Soc., 1996, 118, 2872-2881.

34 (a) M. A. Fox, Photochem. Photobiol., 1990, 52, 617-627; (b) F. Müller and J. Mattay, Chem. Rev., 1993, 93, 99-117; (c) M. Julliard and M. Chanon, Chem. Rev., 1983, 83, 425506; (d) N. Hoffmann, J. Photochem. Photobiol., C, 2008, 9, 43-60; (e) N. A. Romero and D. A. Nicewicz, Chem. Rev., 2016, 116, 10075-10166.

35 F. D. Lewis and R. J. DeVoe, Tetrahedron, 1982, 38, 10691077.

36 For an example of a sensitized intermolecular $[2+2]$ photocycloaddition, see: T. Lei, C. Zhou, M.-Y. Huang, L.-M. Zhao, B. Yang, C. Ye, H. Xiao, Q.-Y. Meng, V. Ramamurthy, C.-H. Tung and L.-Z. Wu, Angew. Chem., Int. Ed., 2017, 56, 15407-15410.

37 For the emission spectrum, see: R. Alonso and T. Bach, Angew. Chem., Int. Ed., 2014, 53, 4368-4371. 